\begin{tabular}{|c|c|}
\hline Title & $\begin{array}{l}\text { Effective resource block allocation procedure for quality of service provisioning in a single operator heterogeneous } \\
\text { LTE-A network }\end{array}$ \\
\hline Author(s) & A sheralieva, A lia; Miyanaga, Y oshikazu \\
\hline Citation & $\begin{array}{l}\text { Computer networks, 108, 1-14 } \\
\text { https://doi.org/10.1016/.comnet.2016.07.012 }\end{array}$ \\
\hline Issue Date & 2016-10-25 \\
\hline Doc URL & http:/hdl.handle.net/2115/71735 \\
\hline Rights & $\begin{array}{l}\text { (2) 2016. This manuscript version is made available under the CC-BY-NC-ND } 4.0 \text { license } \\
\text { http://creativecommons.org/icenses/by-nc-nd/4.0/ }\end{array}$ \\
\hline Rights(URL) & https://creativecommons.org/icenses/by-nc-nd/4.0/ \\
\hline Type & article (author version) \\
\hline File Information & COMNET-D-15-823-DCV.R1.pdf \\
\hline
\end{tabular}

Instructions for use 


\title{
Effective Resource Block Allocation Procedure for Quality of Service Provisioning in a Single- Operator Heterogeneous LTE-A Network
}

\author{
Alia Asheralieva and Yoshikazu Miyanaga
}

\begin{abstract}
We consider the problem of resource block (RB) allocation in the integrated pico/macrocell Long Term Evolution Advanced (LTE-A) network. It is assumed that the network is controlled by a single service provider (SP) and all the operation of the picocells is coordinated with a macro-network. To improve the quality of service (QoS) for end-to-end applications, we take into account the individual traffic demands of the users and allocate the RBs to minimize the sum of user utilities which are expressed in terms of the size of their queues. The formulated RB allocation problem belongs to the family of the multiple knapsack problems (MKPs) and, therefore, it is non-deterministic polynomial time (NP) hard in the strong sense. To reduce the complexity of this problem, we propose a simple heuristic technique to find the suitable (but not necessarily optimal) solution. The proposed RB allocation procedure requires only two additional signalling steps (necessary to maintain the coordination among different cells) and, therefore, its impact of the control signalling overhead is neglectable. It was shown (using OPNET-based simulations) that the proposed technique has low complexity, fast solution time, and shows improved performance when compared to other relevant schemes.
\end{abstract}

Index Terms - Heterogeneous Networks, LTE-A, Resource Allocation.

\section{INTRODUCTION}

$\mathrm{H}$ ETEROGENEOUS network (HetNet) is one of the core features introduced within the third Generation Partnership Project (3GPP) LTE-A standardization framework. In HetNet, the smaller cells (picocells, femtocells, or relay nodes) are served by the lower-power evolved NodeBs (eNBs) [1]. Within the network, the small cells operate on the underlay to the macrocells (by reusing their frequency resources). In this way, the system spectral efficiency can be increased in the hot spot areas with high user demands. On the other hand, the small coverage area of lowerpower eNBs facilitates a rather large number of concurrent transmissions and improves network performance and service quality by offloading traffic from the macrocells [2]. There are many possible ways of practical HetNet realization. For example, the whole network can be operated by a single SP (in which case, the small cells are maintained in coordination with a macro-network). On the other hand, some small cells (e.g., home eNBs) can be privately owned or installed by different operators (with no coordination with a macro-network) [3].

Extensive research on enhancing the performance of LTE-A HetNets has been conducted during the last decade. In heterogeneous networks with a mixture of small cells and macrocells, the interference is a major obstacle that can impair any potential gain of small cells [4]. Consequently, many works have focused on devising the various inter-cell interference coordination (ICIC) methods to alleviate the potential interference in the air interface between the small cells (picocells, femtocells, and relay nodes) and a macronetwork (e.g., [5] for picocells, [6] and [7] for femtocells, [8] and [9] for relay nodes). To further expand the picocell coverage and carry more data traffic, the cell range expansion (CRE) technique (which allows serving the users by the lowpower cells) has been proposed in [10] - [12]. This technique provides the opportunity to share more network load and extend the coverage of the picocells. On the other hand, the discontinuous coverage of the picocells causes the increased registration signalling overhead and decreases the system throughput. This problem has been addressed in [13] where the authors present a new scheme to enhance the performance of a picocell/macrocell network by reducing the signalling overhead related to user location information.

Different spectrum and power allocation strategies to maximize the network throughput have been investigated in [14], [15]. In [14], the authors develop a power allocation technique to maximize the rate of the picocells and show that the proposed scheme outperforms the other sub-optimal power allocation schemes in terms of energy efficiency. The impact of spectrum allocation on the coverage probability $(\mathrm{CP})$ for the picocell users has been analysed in [15]. The proposed framework considers the cases when the macro base stations employ either fractional or soft frequency reuse. Obtained results show that the $\mathrm{CP}$ is maximized when the picocell users operate on the same frequency resources as the central region. Resource allocation in a multi-operator LTE-A HetNet has been investigated in [16]. Here the problem of integrating the picocells with the macrocells owned by different operators is analysed using game theory. The problem is treated as a type of exchange economy. Theoretical performance of the proposed economical model for resource allocation indicates that the total throughput can be improved when both operators allocate the resources to maximize the product of user throughputs.

The brief literature review provided above indicates that there is a growing interest in practical realizations of HetNets. However, there are some critical issues which still remain to 
be enhanced and adapted. First of all, most existing research mainly focus on the connection-layer or physical-layer system parameters, such as signal-to-interference-and-noise ratio (SINR), cell coverage, the number of physical connections and connection dropping probability, physical-layer throughput, and power efficiency. The higher-layer QoS metrics (e.g., endto-end packet delay and loss) have not been considered. Therefore, it is impossible to analyse the actual performance of these methods for end-to-end applications which is crucial for further deployment of HetNets. Secondly, many previous works treat the radio spectrum as a continuous resource. In LTE, however, the spectrum resources are allocated to the users in terms of RBs. The number of RBs varies depending on available bandwidth from $6 \mathrm{RBs}$ (for $1.4 \mathrm{MHz}$ system) up to $100 \mathrm{RBs}$ (for $20 \mathrm{MHz}$ system) but it is still very small to assume the spectrum continuity [17].

In this paper, we propose a novel approach for resource allocation in the integrated picocell/macrocell LTE-A network where the picocells are controlled by a macro-network. We argue that the performance for end-to-end applications could be improved significantly by taking into account the individual traffic demands of the users which can be tracked in LTE systems through the user of the buffer status reporting (BSR) procedure [18]. Subsequently, we express the user utility in terms of the queue size in the buffer of user equipment (UE) and allocate RBs to minimize the sum of user utilities. We choose queue size as a QoS measure because it is directly related to the packet end-to-end delay which determines the higher-layer service performance but rather difficult to estimate (for instance, the end-to-end delay in LTE consists of many delay components, including transmission and queuing delay, propagation and processing delay, the uplink delay due to scheduling and delay due to hybrid automatic repeat request [17]). We show that the formulated $\mathrm{RB}$ allocation problem belongs to the family of the NP-hard MKPs [19]. To reduce the complexity of this problem, we develop a simple heuristic technique to find the suitable (but not necessarily optimal) solution and demonstrate that the proposed $\mathrm{RB}$ allocation procedure achieves the performance close to optimal.

The rest of the paper is organized as follows. The network model is described in Section II. A related optimization problem is formulated in Section III. The proposed heuristic technique is derived in Section IV. The performance of the proposed technique is evaluated in Section V. Results of this work are summarized in Conclusion.

\section{NETWORK MODEL}

The discussion through the rest of the paper (if not stated otherwise) is applicable to both the uplink (UL) and downlink (DL) directions. Consider a heterogeneous LTE-A network (illustrated in Figure 1) that consists of a macro-network comprising one or more conventional eNBs and $N$ picocells served by the low-power pico-eNBs (pNBs). It is assumed that the network is maintained by a single SP and the operation of the picocells is coordinated by the macro-network. Inside the network, the $\mathrm{pNBs}$ are uniquely numbered as $\mathrm{pNB}_{1}, \ldots, \mathrm{pNB}_{N}$.
We use $\mathbf{N}=\{1, \ldots, N\}$ to denote the set of the picocells in our system. The eNBs and pNBs communicate with each other using the standard X2 interface according to the application protocols described in [21]. The network operates on a slottedtime basis with the time axis partitioned into equal nonoverlapping time intervals (slots) of the length $T_{s}$, with $t$ denoting the integer-valued slot index. ${ }^{1}$

The network serves $M$ wireless users, denoted $\mathrm{U}_{1}, \ldots, \mathrm{U}_{M}$, with $\mathbf{M}=\{1, \ldots, M\}$ indicating the set of the users. Note that in LTE, the number of users and their unique users' identification numbers (IDs) can be found from the standard random access channel $(\mathrm{RACH})$ procedure activated at an initial access to the network (i.e., for originating, terminating or registration call) [17]. It is assumed that the coverage areas of the picocells do not overlap which each other but do overlap with the coverage area of a macro-network. Consequently, the users located within the service area of a picocell can be served by this picocell and/or by a macronetwork. The users located outside the service areas of the picocells can be served only by the macro-network. For each $\mathrm{pNB}_{n}$, we use notation $\mathbf{M}_{n}$ and $\mathbf{M}_{0}$ to indicate, respectively, the set of the users placed inside its service area and the set of the users located outside its service area. Clearly, $\mathbf{M}_{n} \subseteq \mathbf{M}$, $n \in \mathbf{N}$, and $\mathbf{M}_{0} \subseteq \mathbf{M}$ represent the partitions of a set $\mathbf{M}$ and, hence,

$\left(\bigcup_{i \in \mathbf{N}} \mathbf{M}_{i}\right) \cup \mathbf{M}_{0}=\mathbf{M}, \mathbf{M}_{n} \cap \mathbf{M}_{0}=\varnothing, \mathbf{M}_{n} \cap \mathbf{M}_{k}=\varnothing, \forall n, k \in \mathbf{N}$.

The considered system operates on the bandwidth spanning $K$ RBs, numbered $\mathrm{RB}_{1}, \ldots, \mathrm{RB}_{K}$, with $\mathbf{K}=\{1, \ldots, K\}$ being the set of all RBs comprising the total available bandwidth. Each eNB/pNB operates on its fixed licensed spectrum band. It is assumed that (i) the spectrum band of the picocells may overlap with the spectrum band of a macro-network but do not overlap with each other; (ii) the frequency reuse within a macro-network is not allowed. By this assumption, the overlapping RBs within the system bandwidth can be occupied by at most two users (a user of a picocell and a user of a macro-network). The rest of the RBs are allocated to at most one user of the eNB or pNB. We use notation $\mathbf{K}_{n}$ and $\mathbf{K}_{0}$ to indicate the set of RBs within the bandwidth of the $\mathrm{pNB}_{n}$ and the set of RBs belonging to the spectrum band of a macronetwork, respectively. Then, the following relations are true:

$\mathbf{K}_{n} \subseteq \mathbf{K}, \mathbf{K}_{0} \subseteq \mathbf{K}, \mathbf{K}_{n} \cap \mathbf{K}_{k}=\varnothing, \forall n, k \in \mathbf{N}$.

Recall that a standard LTE system uses an orthogonal frequency-division multiple access (OFDMA) for DL transmissions and a single carrier frequency division multiple access (SC-FDMA) in the UL direction. Both the SC-FDMA and OFDMA provide the orthogonality of RB allocations to the users served by one cell. Therefore, when information is transmitted by the user to its serving eNB, it will be distorted only by the user(s) associated with some other eNB(s) operating on the same RB(s) [17], [22]. In our model, only the RBs in a shared band $\left(\bigcup_{n \in \mathbf{N}} \mathbf{K}_{n}\right) \cap \mathbf{K}_{0}$ are vulnerable to the

\footnotetext{
${ }^{1}$ This accords to the standard LTE system [1] where the duration of each
} slot $t$ equals $T_{s}=0.5 \mathrm{~ms}$. 
interference. The rest of the spectrum $\mathbf{K} \backslash\left(\bigcup_{n \in \mathbf{N}} \mathbf{K}_{n}\right) \cap \mathbf{K}_{0}$ is free from potential interference.

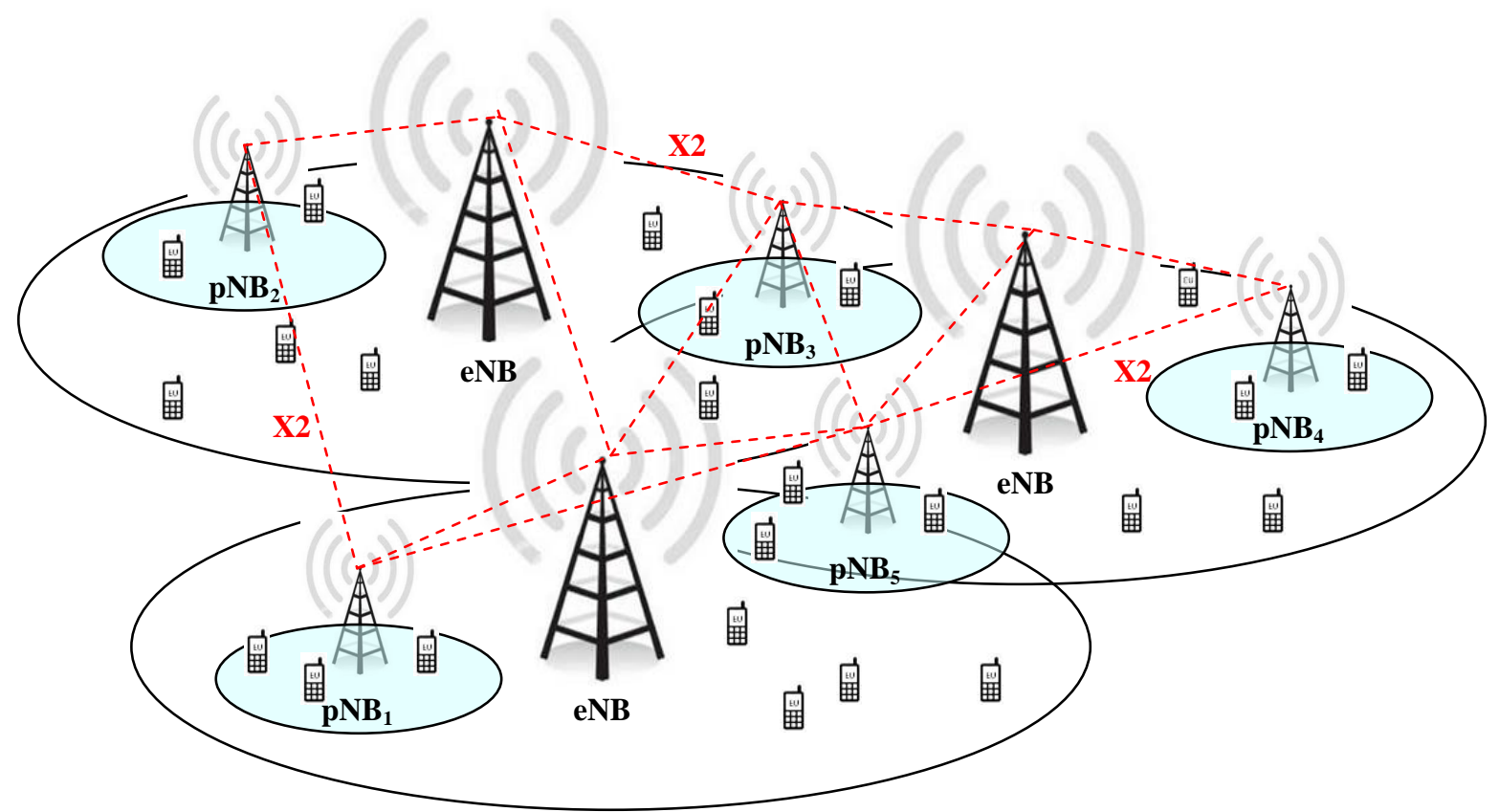

Figure 1. Example of a heterogeneous LTE-A system with the macro-network served by 3 eNBs and 5 picocells served by the pNBs.

\section{PROBLEM StATEMENT}

\section{A. Constraints}

In this Section, we formulate the RB allocation problem for the integrated pico/macro-network described above. First, let us consider the constraints on RB allocations to the users of a macro-network and the picocells in a considered network model. An illustrative example of possible RB allocation is shown in Figure 2. Note that the users located in the picocells can use both the RBs of these picocells (in which case they are served by the $\mathrm{pNB}$ ) and the RBs of a macro-network (in which case they are served by one of the eNBs). On the other hand, the users placed in the non-overlapping (with the picocells) areas can utilize only the RBs of a macro-network. Within the total available bandwidth, each RB in the unprotected (from the interference) spectrum $\left(\bigcup_{n \in \mathbf{N}} \mathbf{K}_{n}\right) \cap \mathbf{K}_{0}$ can be allocated to at most two users: a user of some pNB and a user of a macronetwork. Any RB in the protected (from the interference) spectrum $\mathbf{K} \backslash\left(\bigcup_{n \in \mathbf{N}} \mathbf{K}_{n}\right) \cap \mathbf{K}_{0}$ can be allocated to at most one user (associated with one of the pNBs or eNBs).

Now, for each $n \in \mathbf{N}, m \in \mathbf{M}, k \in \mathbf{K}$, we define the binary variable $x^{m}{ }_{k n}(t)$ equaling 1 , if $\mathrm{RB}_{k}$ is allocated to the user $\mathrm{U}_{m}$ by the $\mathrm{pNB}_{n}$ at slot $t$ and 0 , otherwise. Similarly, for each $m \in \mathbf{M}, k \in \mathbf{K}$, we define the binary variable $y^{m}{ }_{k}(t)$ equaling 1 , if $\mathrm{RB}_{k}$ is allocated to the user $\mathrm{U}_{m}$ by a macro-network. Then, the binary RB allocation variables $x^{m}{ }_{k n}(t)$ and $y^{m}{ }_{k}(t)$ should satisfy the following constraints at any slot $t$ :

$\sum_{m \in \mathbf{M}} x_{k n}^{m}(t) \leq 1, \sum_{m \in \mathbf{M}} y_{k}^{m}(t) \leq 1, \forall k \in \mathbf{K}, \forall n \in \mathbf{N} ;$ $\sum_{m \in \mathbf{M} \backslash \mathbf{M}_{n}} x_{k n}^{m}(t)=0, \forall k \in \mathbf{K}_{n}, \forall n \in \mathbf{N}$

$\sum_{m \in \mathbf{M}_{n}} x_{k n}^{m}(t)=0, \forall k \in \mathbf{K} \backslash \mathbf{K}_{n}, \forall n \in \mathbf{N}$

$\sum_{m \in \mathbf{M}} y_{k}^{m}(t)=0, \forall k \in \mathbf{K} \backslash \mathbf{K}_{0}, \forall n \in \mathbf{N}$;

$\sum_{m \in \mathbf{M}}\left(x_{k n}^{m}(t)+y_{k}^{m}(t)\right) \leq 1, \forall k \in \mathbf{K} \backslash\left(\bigcup_{n \in \mathbf{N}} \mathbf{K}_{n}\right) \cap \mathbf{K}_{0}, \forall n \in \mathbf{N} ;$

$\sum_{m \in \mathbf{M}}\left(x_{k n}^{m}(t)+y_{k}^{m}(t)\right) \leq 2, \forall k \in\left(\bigcup_{n \in \mathbf{N}} \mathbf{K}_{n}\right) \cap \mathbf{K}_{0}, \forall n \in \mathbf{N}$.

In expressions above, the constraint (1a) is necessary to ensure that each RB within the available bandwidth is allocated to at most one user. The constraint (1b) guarantees that the RBs within the spectrum of the $\mathrm{pNB}_{n}$ are allocated only to the users in $\mathbf{M}_{n}$ (i.e., the users placed within the service area of $\mathrm{pNB}_{n}$ ). The constraint (1c) shows that the users in $\mathbf{M}_{n}$ can be allocated only with the RBs in $\mathbf{K}_{n}$ (that is, the RBs in the spectrum of the $\mathrm{pNB}_{n}$ ). The constraint (1d) presumes that a macro-network can allocate only the $\mathrm{RBs}$ in $\mathbf{K}_{0}$. The constraint (1e) shows that the protected RBs can be allocated to at most one user. Finally, the constraint (1f) ensures that the RBs within the shared bandwidth can be allocated to at most two users and one of them should be associated with the pNB, whereas the other one should be served by a macro-network.

Before formulating the optimization problem, we want to add one additional constraint which is necessary to guarantee that the SINRs in the wireless channels between the users and pNBs/eNBs do not fall below some minimal satisfactory levels $\operatorname{SINR}_{\text {tar }}{ }, m \in \mathbf{M}$. Consequently, we assume that any $\mathrm{RB}_{k}$ within the total available spectrum can be allocated to the user $\mathrm{U}_{m}$ only if the following inequalities hold at any slot $t$ : 
$\operatorname{SINR}_{k n}^{m}(t) \geq \operatorname{SINR}_{\text {tar }}^{m}, \operatorname{SINR}_{k 0}^{m}(t) \geq \operatorname{SINR}_{\text {tar }}^{m}$,

for all $n \in \mathbf{N}, m \in \mathbf{M}, k \in \mathbf{K}$. In (2), $\operatorname{SINR}^{m}{ }_{k n}(t)$ is the SINR in the channel between $\mathrm{U}_{m}$ and $\mathrm{pNB}_{n}$ transmitting over the $\mathrm{RB}_{k}$ at slot $t ; \operatorname{SINR}_{k 0}{ }_{k 0}(t)$ is the SINR in the channel between $\mathrm{U}_{m}$ operating on $\mathrm{RB}_{k}$ at slot $t$. In our network model, $\operatorname{SINR}^{m}{ }_{k n}(t)$ can be estimated using

$$
\operatorname{SINR}_{k n}^{m}(t)=\frac{G_{k n}^{m} p^{m}}{\sum_{j \in \mathbf{M}} G_{k n}^{j} p^{j} y_{k}^{j}(t)+\sigma^{2}}, \forall k \in \mathbf{K}, \forall m \in \mathbf{M}, \forall n \in \mathbf{N}
$$

in the UL direction and

$$
\operatorname{SINR}_{k n}^{m}(t)=\frac{G_{k n}^{m} p^{m}}{\sum_{j \in \mathbf{M}} G_{k 0}^{m} p^{j} y_{k}^{j}(t)+\sigma^{2}}, \forall k \in \mathbf{K}, \forall m \in \mathbf{M}, \forall n \in \mathbf{N}
$$

in the DL direction. In (3a) and (3b), $G^{m}{ }_{k n}$ is the link gain of the channel between $\mathrm{U}_{m}$ and $\mathrm{pNB}_{n}$ transmitting over $\mathrm{RB}_{k}, G^{m}{ }_{k 0}$ is the link gain of the channel between $\mathrm{U}_{m}$ operating on $\mathrm{RB}_{k}$ and a macro-network, $\sigma^{2}$ is the Additive white Gaussian noise (AWGN) power, $p^{m}$ is the transmission power in the (UL or DL) channel between $\mathrm{U}_{m}$ and its associated $\mathrm{eNB} / \mathrm{pNB}$.

On the other hand, $\operatorname{SINR}_{k 0}^{m}(t)$ can be calculated using

$$
\operatorname{SINR}_{k 0}^{m}(t)=\frac{G_{k 0}^{m} p^{m}}{\sum_{i \in \mathbf{N}} \sum_{j \in \mathbf{M}} G_{k 0}^{j} p^{j} x_{k i}^{j}(t)+\sigma^{2}}, \forall k \in \mathbf{K}, \forall m \in \mathbf{M}
$$

in the UL direction and

$$
\operatorname{SINR}_{k 0}^{m}(t)=\frac{G_{k 0}^{m} p^{m}}{\sum_{i \in \mathbf{N}} \sum_{j \in \mathbf{M}} G_{k i}^{m} p^{j} x_{k i}^{j}(t)+\sigma^{2}}, \forall k \in \mathbf{K}, \forall m \in \mathbf{M}
$$

in the DL direction. Note that in LTE, the instantaneous values of $G^{m}{ }_{k n}$ and $G^{m}{ }_{k 0}$ can be obtained from the channel state information (CSI), for all $n \in \mathbf{N}, m \in \mathbf{M}, k \in \mathbf{K}$, through the use of the standard reference signals (RSs) [22], [23]. This means that at any slot $t$, we rely on the global knowledge of the accurate CSI in all wireless channels. The transmission power levels $p^{m}$ are assumed to be fixed and known to all the eNBs/pNBs, for all $m \in \mathbf{M}$. Indeed, the design of a standard LTE system provides the possibility of collecting the above information [17], [22], [23]. However, in any network operating in a "real-world" environment, some data may not be available for the certain wireless channel at certain time instants. Based on such considerations, further in the paper, we assume that the values of $G^{m}{ }_{k n}$ and $G^{m}{ }_{k 0}$ are obtained from the last available CSI.

\section{B. Optimization Problem}

Any resource allocation algorithm is usually designed to exploit the varying network characteristics by adaptively distributing scarce communication resources to optimize some certain performance metrics. The goal of resource allocation in our network model is to utilize all available RBs to improve the end-to-end QoS for the network users. Consequently, in order to design effective RB allocation algorithm, it is very important to choose an appropriate system parameter to measure the service performance. The existing research on resource allocation in LTE-A HetNets ([5] - [15]) mainly focus on the reliability of wireless communications by optimizing such physical (PHY) layer system parameters as
SINR or PHY-layer throughput. Communication reliability is a very important aspect of the network performance but not enough to consider for improving the user-perceived QoS for nowadays applications (such as live streaming, online games, and video sharing). It is, therefore, equally important to take into account the individual traffic demands of the users in order to improve the higher-layer service performance metrics (e.g., packet delay).

In LTE, the instantaneous data on the arrived and enqueued traffic is readily available through the use of the BSR procedure (described, in detail, in [18]). As part of packet scheduling, at the beginning of each slot $t$, each user $\mathrm{U}_{m}$ is required to send its instantaneous buffer status information (bit arrival rate $A^{m}(t)$ and buffer size in bits $Q^{m}(t)$ ) via a dedicated physical uplink control channel (PUCCH). In this way, the eNBs/pNBs get to "know" the exact amount of the UL data arrived and enqueued in the buffers of UEs. After receiving the BSRs from all associated UEs, the eNBs/pNBs allocate RBs and send the allocation information to the corresponding users via physical downlink control channels (PDCCHs). Similarly, the eNB readily finds out the size of the DL traffic arrived and enqueued in the buffer of each UE. The available instantaneous information on the arrived and enqueued traffic, together with the number of RBs allocated to each user, can be used to calculate the future queue size for each UE. For this, it is enough to apply the well-known Lindley's equation [24]

$Q^{m}(t+1)=\left\lceil Q^{m}(t)+A^{m}(t)-R^{m}(t)\right\rceil^{+}, \forall m \in \mathbf{M}$

where $\lceil X\rceil^{+}=\max (0, X) ; Q^{m}(t), A^{m}(t), R^{m}(t)$ are, respectively, the size of the queue (in bits), the bit arrival rate (in bps), and the bit service rate (in bps) in the channel of $\mathrm{U}_{m}$ at slot $t$.

In (5), the exact values of $Q^{m}(t)$ and $A^{m}(t)$ are transmitted to the eNBs/pNBs during the BSR procedure. The value of $R^{m}(t)$ depends on the number of RBs allocated to user $\mathrm{U}_{m}$ at time slot $t$ and their transmission rates (which depends on the instantaneous SINR over these RBs). In our network model, $R^{m}(t)$ can be calculated using

$$
\begin{aligned}
& R^{m}(t)=\sum_{k \in \mathbf{K}}\left[y_{k}^{m}(t) r_{k 0}^{m}(t)+\sum_{n \in \mathbf{N}} x_{k n}^{m}(t) r_{k n}^{m}(t)\right]= \\
& =\frac{\omega}{T_{s}} \sum_{k \in \mathbf{K}}\left[y_{k}^{m}(t) \log \left(1+\operatorname{SINR}_{k 0}^{m}(t)\right)+\sum_{n \in \mathbf{N}} x_{k n}^{m}(t) \log \left(1+\operatorname{SINR}_{k n}^{m}(t)\right)\right],
\end{aligned}
$$

for all $m \in \mathbf{M}$. In (6), $\omega=180 \mathrm{kHz}$ is the bandwidth of one RB, $T_{s}=1 \mathrm{~ms}$ is the duration of one RB; $r^{m}{ }_{k 0}(t)$ is the service rate at slot $t$ in the channel between $\mathrm{U}_{m}$ operating on $\mathrm{RB}_{k}$ and a macro-network, $r^{m}{ }_{k n}(t)$ is the service rate at slot $t$ in the channel between $\mathrm{U}_{m}$ transmitting over $\mathrm{RB}_{k}$ and the $\mathrm{pNB}_{n}$.

Further in the paper, the future queue size will be used as a QoS measure for each UE. The rationales behind this particular choice are as follows:

- At any slot $t$, the future queue size $Q^{m}(t+1)$ of $\mathrm{U}_{m}$ can be easily estimated using the Lindley's equation (5), for all $m \in \mathbf{M}$.

- The queue size is directly related to the packet delay in the buffers of UEs.

Note that to minimize the packet delay in the buffer of $\mathrm{U}_{m}$, we 
have to minimize its future queue size $Q^{m}(t+1)$ (by serving the maximal possible amount of data arrived and enqueued in the buffer of UE at any slot $t$ ). However, if we simply maximize the service rate $R^{m}(t)$, for all $m \in \mathbf{M}$, then we may arrive at a situation when $R^{m}(t) \geq Q^{m}(t)+A^{m}(t)$, for one or more $m \in \mathbf{M}$. This means that some of the network resources (counted in terms of RBs in our system) will be wasted on increasing the service rate for the users with smaller traffic demands. In order to avoid this situation, instead of maximizing the current service rate $R^{m}(t)$ we propose to minimize the future queue size $Q^{m}(t+1)$.

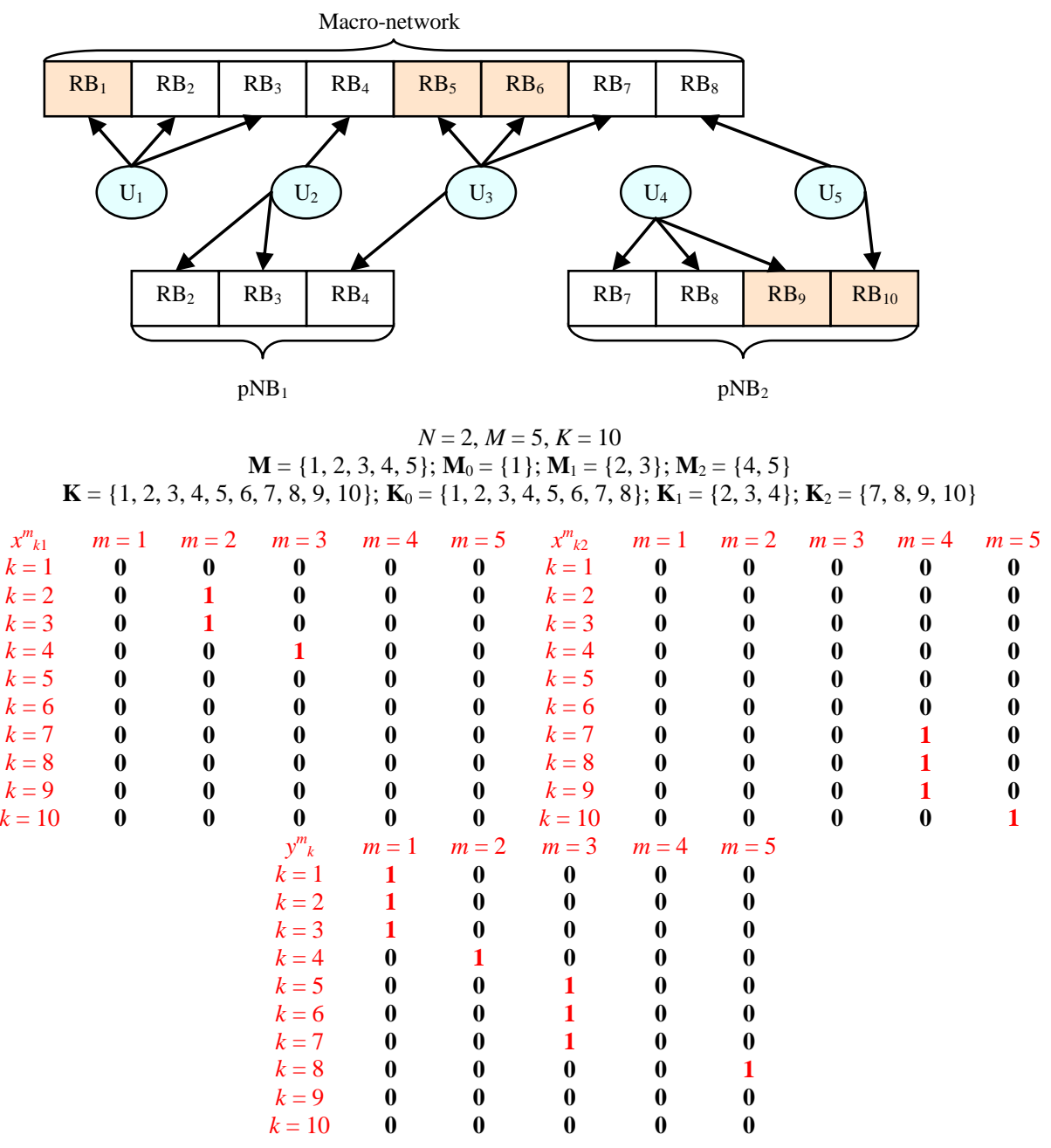

Figure 2. Example of RB allocation in the network with 2 pNBs and 5 users.

With the constraints on the binary RB allocation variable $x^{m}{ }_{k n}(t)$ and $y^{m}{ }_{k}(t)$, given by (1a) - (1f), the target SINR constraints in (2), and the objective function defined in (5), we are ready to formulate the RB allocation problem (to simplify notation, we skip the index $t$ below and further in the paper):

minimize $\sum_{m \in \mathbf{M}} \theta^{m}\left\lceil Q^{m}+A^{m}-R^{m}\right\rceil^{+}$

subject to:

$$
\begin{aligned}
& x_{k n}^{m} \in\{0,1\}, y_{k}^{m} \in\{0,1\}, \forall k \in \mathbf{K}, \forall m \in \mathbf{M}, \forall n \in \mathbf{N} ; \\
& \sum_{m \in \mathbf{M}} x_{k n}^{m} \leq 1, \sum_{m \in \mathbf{M}} y_{k}^{m} \leq 1, \forall k \in \mathbf{K}, \forall n \in \mathbf{N} ; \\
& \sum_{m \in \mathbf{M} \backslash \mathbf{M}_{n}} x_{k n}^{m}=0, \forall k \in \mathbf{K}_{n}, \forall n \in \mathbf{N} ;
\end{aligned}
$$

$$
\begin{aligned}
& \sum_{m \in \mathbf{M}_{n}} x_{k n}^{m}=0, \forall k \in \mathbf{K} \backslash \mathbf{K}_{n}, \forall n \in \mathbf{N} ; \\
& \sum_{m \in \mathbf{M}} y_{k}^{m}=0, \forall k \in \mathbf{K} \backslash \mathbf{K}_{0}, \forall n \in \mathbf{N} ; \\
& \sum_{m \in \mathbf{M}}\left(x_{k n}^{m}+y_{k}^{m}\right) \leq 1, \forall k \in \mathbf{K} \backslash\left(\bigcup_{n \in \mathbf{N}} \mathbf{K}_{n}\right) \cap \mathbf{K}_{0}, \forall n \in \mathbf{N} ; \\
& \sum_{m \in \mathbf{M}}\left(x_{k n}^{m}+y_{k}^{m}\right) \leq 2, \forall k \in\left(\bigcup_{n \in \mathbf{N}} \mathbf{K}_{n}\right) \cap \mathbf{K}_{0}, \forall n \in \mathbf{N} ; \\
& \operatorname{SINR}_{k n}^{m} \geq \operatorname{SINR} R_{t a r}^{m}, \forall k \in \mathbf{K}, \forall m \in \mathbf{M}, \forall n \in \mathbf{N} ; \\
& \operatorname{SINR}_{k 0}^{m} \geq \operatorname{SINR} R_{t a r}^{m}, \forall k \in \mathbf{K}, \forall m \in \mathbf{M},
\end{aligned}
$$

where $\theta^{m} \in(0,1]$ is the "weight" of $\mathrm{U}_{m}$ which is used to prioritize the users according to their QoS requirements (e.g., for real-time applications with strict delay requirements, $\theta^{m}$ could be set equal 1, whereas, for the "best-effort" users, it 
could be assigned any positive value, smaller than 1). In (7a) (7j), the parameters $\theta^{m}, Q^{m}, A^{m}$, and $\operatorname{SINR}^{m}{ }_{\text {tar }}$ are known at any slot $t$, for all $m \in \mathbf{M}$. The service rates $R^{m}$ (given by (7)) and the SINR levels $\operatorname{SINR}^{m}{ }_{k n}$ (given by (3a) for the UL direction and (3b) for the DL direction) and $\operatorname{SINR}_{k 0}^{m}$ (given by (4a) for the UL direction and (4b) for the DL direction) are the functions of the binary optimization variables $x^{m}{ }_{k n}$ and $y^{m}{ }_{k}$.

\section{RB ALLOCATION PROCEDURE}

Note that the objective in (7a) can be minimized by maximizing the weighted sum of service rates, $\sum_{m \in \mathbf{M}} \theta^{m} R^{m}$

(since $Q^{m}$ and $A^{m}$ do are some constants which do not depend on the optimization variable $x^{m}{ }_{k n}$ and $y^{m}{ }_{k}$ ). However, because of the operator [\rceil$^{+}$that places the non-negativity restrictions on the future queue size $Q^{m}+A^{m}-R^{m}$, we have to constraint the service rate $R^{m}$, for each $m \in \mathbf{M}$, to stay below the sum $Q^{m}$ $+A^{m}$. Consequently, the optimization objective (7a) can be stated, alternatively, as

$$
\text { minimize } \sum_{m \in \mathbf{M}} \theta^{m} R^{m}
$$

subject to: $R^{m} \leq Q^{m}+A^{m}, \forall m \in \mathbf{M}$.

Based on (8a) and (8b), we formulate the optimization problem $(7 \mathrm{a})-(7 \mathrm{j})$, as

$$
\text { minimize } \sum_{m \in \mathbf{M}} \theta^{m} \sum_{k \in \mathbf{K}}\left(y_{k}^{m} r_{k 0}^{m}+\sum_{n \in \mathbf{N}} x_{k n}^{m} r_{k n}^{m}\right)
$$

subject to:

$$
\begin{aligned}
& x_{k n}^{m} \in\{0,1\}, y_{k}^{m} \in\{0,1\}, \forall k \in \mathbf{K}, \forall m \in \mathbf{M}, \forall n \in \mathbf{N} ; \\
& \sum_{m \in \mathbf{M}} x_{k n}^{m} \leq 1, \sum_{m \in \mathbf{M}} y_{k}^{m} \leq 1, \forall k \in \mathbf{K}, \forall n \in \mathbf{N} ; \\
& \sum_{m \in \mathbf{M} \backslash \mathbf{M}_{n}} x_{k n}^{m}=0, \forall k \in \mathbf{K}_{n}, \forall n \in \mathbf{N} ; \\
& \sum_{m \in \mathbf{M}_{n}} x_{k n}^{m}=0, \forall k \in \mathbf{K} \backslash \mathbf{K}_{n}, \forall n \in \mathbf{N} ; \\
& \sum_{m \in \mathbf{M}} y_{k}^{m}=0, \forall k \in \mathbf{K} \backslash \mathbf{K}_{0}, \forall n \in \mathbf{N} ; \\
& \sum_{m \in \mathbf{M}}\left(x_{k n}^{m}+y_{k}^{m}\right) \leq 1, \forall k \in \mathbf{K} \backslash\left(\bigcup_{n \in \mathbf{N}} \mathbf{K}_{n}\right) \cap \mathbf{K}_{0}, \forall n \in \mathbf{N} ; \\
& \sum_{m \in \mathbf{M}}\left(x_{k n}^{m}+y_{k}^{m}\right) \leq 2, \forall k \in\left(\bigcup_{n \in \mathbf{N}} \mathbf{K}_{n}\right) \cap \mathbf{K}_{0}, \forall n \in \mathbf{N} ; \\
& S I N R_{k n}^{m} \geq S I N R_{t a r}^{m}, \forall k \in \mathbf{K}, \forall m \in \mathbf{M}, \forall n \in \mathbf{N} ; \\
& S I N R_{k 0}^{m} \geq S I N R_{t a r}^{m}, \forall k \in \mathbf{K}, \forall m \in \mathbf{M} ; \\
& \sum_{k \in \mathbf{K}}\left(y_{k}^{m} r_{k 0}^{m}+\sum_{n \in \mathbf{N}} x_{k n}^{m} r_{k n}^{m}\right) \leq Q^{m}+A^{m}, \forall m \in \mathbf{M} \\
& W
\end{aligned}
$$

where the rates $R^{m}$ are expressed (as in (6)) using the transmission rates of RBs, $r^{m}{ }_{k 0}$ and $r^{m}{ }_{k n}$.

Apparently, the above problem belongs to the family of MKPs [19]. In our variant of the problem, the set of $K$ items (RBs), denoted by $\mathbf{K}$, is partitioned into $N+1$ different subsets or classes (indicating the RB association with a macronetwork or pNB), $\mathbf{K}_{0}, \mathbf{K}_{1}, \ldots, \mathbf{K}_{N}$. Similarly, the set of $M$ knapsacks (users), denoted by $\mathbf{M}$, is partitioned into $N+1$ different classes (indicating user association with a macronetwork or $\mathrm{pNB}), \mathbf{M}_{0}, \mathbf{M}_{1}, \ldots, \mathbf{M}_{N}$. Each $k^{\text {th }}$ item has the maximal profit (or weight) $\sum_{m \in \mathbf{M}} \theta^{m} r_{k 0}^{m} \geq 0$, if it belongs to class 0 , and $\sum_{m \in \mathbf{M}} \theta^{m} r_{k n}^{m} \geq 0$, if it is in class $n \in \mathbf{N}$. Each $m^{\text {th }}$ knapsack has the capacity $\theta^{m}\left(Q^{m}+A^{m}\right) \geq 0, m \in \mathbf{M}$. The binary variable $y^{m}{ }_{k}$ indicates whether the $k^{\text {th }}$ item belonging to class 0 is placed into the $m^{\text {th }}$ knapsack (in which case, $y^{m}{ }_{k}=1$ and $y^{m}{ }_{k}=0$, otherwise). On the other hand, $x^{m}{ }_{k n}=1$ shows that the $m^{\text {th }}$ knapsack contains the $k^{\text {th }}$ item of a class $n$ (otherwise, $x^{m}{ }_{k n}=$ $0)$. The constraints $(9 c)-(9 f)$ indicate restrictions on the choice of the items and knapsacks belonging to different classes imposed by the considered network model. The constraints $(9 \mathrm{~g})$ and $(9 \mathrm{~h})$ show that the items from the set $\mathbf{K} \backslash\left(\bigcup_{n \in \mathbf{N}} \mathbf{K}_{n}\right) \cap \mathbf{K}_{0}$ can be packed only once, while the items in $\left(\bigcup_{n \in \mathbf{N}} \mathbf{K}_{n}\right) \cap \mathbf{K}_{0}$ can be placed twice to different knapsacks. The objective of this MKP is to pack the items to the knapsacks in the way maximizing the total profit.

All MKPs are NP-hard in the strong sense (the NP-hardness proof for MLPs is provided in [25]). This means that it is not possible to find a fully polynomial approximation scheme for such problems. On the other hand, any dynamic programming approach would result in strictly exponential time bounds [19]. Up to now, there exist two main approaches to deal with large MKPs: i) speed up the solution process using various branchand-bound (B\&B) techniques (e.g., [19], [26] - [28]); ii) find good but not necessarily optimal solutions using heuristic algorithms (e.g., [29] - [32]). In this paper, both of the above approaches are used to deal with the problem (9). To find the optimal solution of the problem, we use the version of an exact B\&B algorithm proposed in [28] for large MKP instances. A comprehensive description of this algorithm can be found in [28]. In short, the algorithm uses a surrogate relaxation for deriving upper bounds to fathom the nodes that cannot lead to an improved solution. The main reason behind this particular choice of the algorithm is its very short solution time for MKPs with a large number of items [19], [28].

Among many heuristic methods for solving large MKPs that have been developed in the past, the most prominent and simple is a so-called greedy allocation (GA) strategy [33]. The GA approach (with some modifications) can also be applied to our problem. In our case, the GA strategy is to ensure that the transmission resources of the network are fully utilized its users. This means that we will prefer such allocations for which the absolute difference $\left|\theta^{m}\left(Q^{m}+A^{m}-r_{k 0}^{m}\right)\right|$, for $m \in \mathbf{M}_{0}$ or $\left|\theta^{m}\left(Q^{m}+A^{m}-r_{k n}^{m}\right)\right|$, for $m \in \mathbf{M}_{n}, n \in \mathbf{N}$, is as small as possible. ${ }^{2}$ Consequently, at each step, we select the user $\mathrm{U}_{i}$, such that

\footnotetext{
${ }^{2}$ Note that the proposed resource allocation approach is similar to the one used in backpressure routing [34]. However, unlike conventional backpressure algorithms, the allocated resources and the users in our problem belong to different classes ( $\mathbf{K}_{0}, \mathbf{K}_{1}, \ldots, \mathbf{K}_{N}$ and $\mathbf{M}_{0}, \mathbf{M}_{1}, \ldots, \mathbf{M}_{N}$, respectively) that are characterized by different features (following from the corresponding constraints $(9 \mathrm{c})-(9 \mathrm{~h})$ describing the relations between various subsets of users and RBs). Such limitations on RB allocations (together with the additional target SINR constraints and positivity requirements in (9i) - (9k))
} 


$$
i \leftarrow \underset{m \in \mathbf{M}}{\arg \max } \theta^{m}\left(Q^{m}+A^{m}\right)
$$

and allocate this user with the best matching $\mathrm{RB}_{j}$, satisfying $j \leftarrow \arg \min \left(\min _{k \in \mathbf{K}_{n}}\left|\theta^{i}\left(Q^{i}+A^{i}-r_{k n}^{i}\right)\right|, \min _{k \in \mathbf{K}_{0}}\left|\theta^{i}\left(Q^{i}+A^{i}-r_{k 0}^{i}\right)\right|\right)$,

if $i \in \mathbf{M}_{n}, n \in \mathbf{N}$, and

$j \leftarrow \underset{k \in \mathbf{K}_{0}}{\arg \min }\left|\theta^{i}\left(Q^{i}+A^{i}-r_{k 0}^{i}\right)\right|$,

otherwise. In $(10 \mathrm{a})-(10 \mathrm{c})$, the values of $\theta^{m}$ are predetermined in the network (based on user application), $Q^{m}, A^{m}$ are known (from the BSRs sent by the users), for all $m \in \mathbf{M}$, at any slot $t$. On the other hand, the values of $r^{m}{ }_{k n}$ and $r^{m}{ }_{k 0}$ depend on the current RB allocations $x^{m}{ }_{k n}$ and $y^{m}{ }_{k}$ and, hence, cannot be known in advance. However, given the past (last available) CSI observations, we can (roughly) estimate these values at any slot $t$, using

$\hat{r}_{k 0}^{m}=\frac{\omega}{T_{s}} \log \left(1+\operatorname{SINR}_{k 0}^{m}(t-1)\right), \hat{r}_{k n}^{m}=\frac{\omega}{T_{s}} \log \left(1+\operatorname{SINR}_{k n}^{m}(t-1)\right)$

for all $m \in \mathbf{M}, k \in \mathbf{K}, n \in \mathbf{N}$.

The proposed algorithm for RB allocation algorithm (outlined, in detail, in Figure 3), is repeated, at each slot $t$, after collecting all data (such as BSR and CSI) by the macronetwork, as summarized below:

Lines 0 and 1. Input all available data.

Line 2. Initialize the variables. Here we create the temporary sets $\hat{\mathbf{M}}, \hat{\mathbf{M}}_{0}, \hat{\mathbf{M}}_{n}, \hat{\mathbf{K}}, \hat{\mathbf{K}}_{0}, \hat{\mathbf{K}}_{n}$ and variables $v^{m}, u_{k}$ and initialize the binary variables $x^{m}{ }_{k n}, y^{m}{ }_{k}$ for all $n \in \mathbf{N}, m \in \mathbf{M}, k \in \mathbf{K}$. The temporary sets $\hat{\mathbf{M}} \subseteq \mathbf{M}, \hat{\mathbf{M}}_{0} \subseteq \mathbf{M}_{0}, \hat{\mathbf{M}}_{n} \subseteq \mathbf{M}_{n}$ are used to store the indices of all network users which need RBs to be allocated (set $\hat{\mathbf{M}}_{0}$ contains only the indices of the users located inside the non-overlapping areas of a macro-network, set $\hat{\mathbf{M}}_{n}$ comprises the indices of the users placed inside the service area of $\mathrm{pNB}_{n}$ ). Similarly, the temporary sets $\hat{\mathbf{K}} \subseteq \mathbf{K}$, $\hat{\mathbf{K}}_{0} \subseteq \mathbf{K}_{0}, \hat{\mathbf{K}}_{n} \subseteq \mathbf{K}_{n}$ contain the RBs that can be allocated to the users $\left(\hat{\mathbf{K}}_{0}\right.$ stores only the RBs within the spectrum of a macro-network, $\hat{\mathbf{K}}_{n}$ comprises the RBs in the band of $\mathrm{pNB}_{n}$ ). The variable $v^{m}$ is used to track the buffer size of $\mathrm{U}_{m}$ during $\mathrm{RB}$ allocation procedure, $u_{k}$ indicates how many times a given $\mathrm{RB}_{k}$ has been allocated.

Lines 3 - 13. Form the temporary sets $\hat{\mathbf{K}}, \hat{\mathbf{K}}_{0}, \hat{\mathbf{K}}_{n}$ containing the indices of RBs which meet the target SINR requirements and estimate the transmission rate of each RB.

Line 14. Start the main loop. Perform RB allocation while both of the sets, $\hat{\mathbf{M}}$ and $\hat{\mathbf{K}}$, are non-empty.

Line 15. Find the user with maximal weighted buffer size $\theta^{i} v^{i}$.

Line 16. If $\mathrm{U}_{i}$ is located within the non-overlapping areas of a macro-network, perform operations in Lines 17 - 24. Else, go to Line 25.

introduce the additional complexity and bring a certain novelty to our RB allocation method.
Line 17. If the set $\hat{\mathbf{K}}_{0}$ is non-empty (and there is at least one $\mathrm{RB}$ that can be allocated to $\mathrm{U}_{i}$ ), perform operations in Lines 18 - 23 else go to Line 24.

Line 18. Find the best matching $R B$ with the minimal difference $\left|\theta^{i}\left(v^{i}-\hat{r}_{k 0}^{m}\right)\right|$ within the set $\hat{\mathbf{K}}_{0}$. Store the index of the best matching $\mathrm{RB}$ in temporary variable $j$. Update (increment by one) the binary variable $y_{j}^{i}$ to show that $\mathrm{RB}_{j}$ is allocated to $\mathrm{U}_{i}$ by a macro-network.

Line 19. Update (decrement by $\hat{r}_{k 0}^{m}$ or reduce to zero) the variable $v^{i}$ representing the buffer size of $\mathrm{U}_{i}$ after $\mathrm{RB}$ allocation.

Line 20. Update (increment by one) the variable $u_{j}$ indicating how many times $\mathrm{RB}_{j}$ has been allocated.

Line 21. Remove $\mathrm{RB}_{j}$ from $\hat{\mathbf{K}}_{0}$.

Line 22. If $v^{i}=0$ then $\mathrm{U}_{i}$ does not need any $\mathrm{RBs}$ to be allocated and we remove it from the set $\hat{\mathbf{M}}$.

Line 23. If $\mathrm{RB}_{j}$ belongs to the set of protected RBs or it has already been allocated more than once $\left(u_{j}>1\right)$, then $\mathrm{RB}_{j}$ cannot be used any longer and we remove it from $\hat{\mathbf{K}}$.

Line 24. If set $\hat{\mathbf{K}}_{0}$ is empty, then there are no RBs that can be allocated to $\mathrm{U}_{i}$, and we remove it from the set $\hat{\mathbf{M}}$.

Line 25. If $\mathrm{U}_{i}$ is located inside the service area of one of the pNBs, perform operations in Lines 26 - 35.

Lines 26 and 27. Find subset $\mathbf{M}_{n}$, such that $i \in \mathbf{M}_{n}$.

Line 28. If the union $\hat{\mathbf{K}}_{0} \cup \hat{\mathbf{K}}_{n}$ is non-empty (that is, there is at least one $\mathrm{RB}$ that can be allocated to $\mathrm{U}_{i}$ ), perform operations in Lines 29 - 34. Else, go to Line 35.

Line 29. Find the best matching $\mathrm{RB}$ from the set $\hat{\mathbf{K}}_{0} \cup \hat{\mathbf{K}}_{n}$ with minimal difference $\left|\theta^{i}\left(v^{i}-\hat{r}_{k n}^{m}\right)\right|$ or $\left|\theta^{i}\left(v^{i}-\hat{r}_{k 0}^{m}\right)\right|$. Store the index of the best matching RB in temporary variable $j$.

Line 30. If $j \in \hat{\mathbf{K}}_{0}$, update (increment by one) the binary variable $y_{j}^{i}$ (showing that $\mathrm{RB}_{j}$ is allocated to $\mathrm{U}_{i}$ by a macronetwork). Remove $\mathrm{RB}_{j}$ from the set $\hat{\mathbf{K}}_{0}$. Update (decrement by $\hat{r}_{k 0}^{m}$ or reduce to zero) the variable $v^{i}$ representing the buffer size of $\mathrm{U}_{i}$ after $\mathrm{RB}$ allocation.

Line 31. Else (if $j \in \hat{\mathbf{K}}_{n}$ ), update (increment by one) the binary $x_{j n}^{i}$ showing that $\mathrm{RB}_{j}$ is allocated to $\mathrm{U}_{i}$ by the $\mathrm{pNB}_{n}$. Remove $\mathrm{RB}_{j}$ from the set $\hat{\mathbf{K}}_{n}$. Update (decrement by $\hat{r}_{k n}^{m}$ or reduce to zero) the variable $v^{i}$ representing the buffer size of $\mathrm{U}_{i}$ after RB allocation.

Line 32. Update (increment by one) the variable $u_{j}$ indicating how many times the $\mathrm{RB}_{j}$ has been allocated.Line 33. If $v^{i}=0$, then $\mathrm{U}_{i}$ does not need any RBs to be allocated and we remove it from the set $\hat{\mathbf{M}}$.

Line 34. If $\mathrm{RB}_{j}$ belongs to the set of protected $\mathrm{RBs}$ or it has already been allocated more than once, then $\mathrm{RB}_{j}$ cannot be used any longer and we remove it from the set $\hat{\mathbf{K}}$.

Line 35. If $\hat{\mathbf{K}}_{0} \cup \hat{\mathbf{K}}_{n}$ is empty, then there are no RBs that can be allocated to $U_{i}$ and we remove it from the set $\hat{\mathbf{M}}$.

Lines 36. Output the results $x_{k n}^{m}, y_{k}^{m}$, for all $m \in \mathbf{M}, k \in \mathbf{K}$. 
0. Given $\mathbf{N}, \mathbf{M}, \mathbf{K}, \mathbf{M}_{0}, \mathbf{K}_{0}, \mathbf{M}_{n}, \mathbf{K}_{n}$, for all $n \in \mathbf{N} ; \quad / *$ Input available data.*/

1. Input $\theta^{m}, Q^{m}+A^{m}, \operatorname{SINR}^{m}{ }_{t a r}, \operatorname{SINR}^{m}{ }_{k 0}(t-1), \operatorname{SINR}_{k n}{ }_{k n}(t-1)$, for all $n \in \mathbf{N}, m \in \mathbf{M}, k \in \mathbf{K}$;

2. Initialize $\hat{\mathbf{M}} \leftarrow \mathbf{M}, \hat{\mathbf{K}} \leftarrow \varnothing, \hat{\mathbf{K}}_{0} \leftarrow \varnothing, \hat{\mathbf{K}}_{n} \leftarrow \varnothing, v^{m} \leftarrow Q^{m}+A^{m}, u_{k} \leftarrow 0, x^{m}{ }_{k n} \leftarrow 0, y^{m}{ }_{k} \leftarrow 0$, for all $n \in \mathbf{N}, m \in \mathbf{M}, k \in \mathbf{K} ; / *$ Initialize temporary sets and variables. $* /$

/*Estimate the transmission rate of all RBs, identify the RBs satisfying the target SINR requirements and put them to the sets

$\hat{\mathbf{K}}, \hat{\mathbf{K}}_{0}, \hat{\mathbf{K}}_{n} *$ /

3. For all $m \in \mathbf{M}$ do \{

4. For all $k \in \mathbf{K}_{0}$ do \{

5. $\quad$ Set $\hat{r}_{k 0}^{m} \leftarrow \omega \log \left(1+\operatorname{SINR}_{k 0}^{m}(t-1)\right) / T_{s}$;

6. If $\left(\operatorname{SINR}^{m}{ }_{k 0}(t-1) \geq \operatorname{SINR}^{m}{ }_{t a r}\right)\{$

7. $\quad$ set $\left.\left.\left.\hat{\mathbf{K}} \leftarrow \hat{\mathbf{K}} \cup\{k\}, \hat{\mathbf{K}}_{0} \leftarrow \hat{\mathbf{K}}_{0} \cup\{k\} ;\right\}\right\}\right\}$

8. For all $n \in \mathbf{N}$ do \{

9. For all $m \in \mathbf{M}$ do \{

10. For all $k \in \mathbf{K}$ do \{

11. $\quad$ Set $\hat{r}_{k n}^{m} \leftarrow \omega \log \left(1+\operatorname{SINR}_{k n}^{m}(t-1)\right) / T_{s}$;

12. If $\left(\operatorname{SINR}_{k n}^{m}(t-1) \geq \operatorname{SINR}_{\text {tar }}^{m}\right)\{$

13. $\quad$ set $\left.\left.\left.\left.\hat{\mathbf{K}} \leftarrow \hat{\mathbf{K}} \cup\{k\}, \hat{\mathbf{K}}_{n} \leftarrow \hat{\mathbf{K}}_{n} \cup\{k\} ;\right\}\right\}\right\}\right\}$

/*While $\hat{\mathbf{M}}$ and $\hat{\mathbf{K}}$ are non-empty, perform RB allocation.*/

14. While $((\hat{\mathbf{M}} \neq \varnothing)$ and $(\hat{\mathbf{K}} \neq \varnothing))\{$

15. Set $i \leftarrow \underset{m \in \hat{\mathbf{M}}}{\arg \max } \theta^{m} v^{m} ; / *$ find the user with maximal weighted buffer size $* /$

16. If $\left(i \in \mathbf{M}_{0}\right)\left\{/ *\right.$ if $\mathrm{U}_{i}$ is located beyond the cells of pNBs, he can access only the spectrum of a macro-network*/

17. If $\left(\hat{\mathbf{K}}_{0} \neq \varnothing\right)\left\{\right.$ I*If $\hat{\mathbf{K}}_{0}$ is non-empty*/

18. Set $j \leftarrow \arg \min \left|\theta^{i}\left(v^{i}-\hat{r}_{k 0}^{i}\right)\right|, y_{j}^{i} \leftarrow y_{j}^{i}+1 ;{ }^{*}$ allocate the best matching RB from $\hat{\mathbf{K}}_{0}$ to $\mathrm{U}_{i}^{*} /$

19. Set $v^{i} \leftarrow\left\lceil v^{i}-\hat{r}_{j 0}^{i}\right\rceil^{+} ; / *$ update $v^{i}$ representing the buffer size of $\mathrm{U}_{i}$ after RB allocation*/

20. Set $u_{j} \leftarrow u_{j}+1 ; / *$ update $u_{j}$ indicating how many times the $\mathrm{RB}_{j}$ has been allocated */

21. Set $\hat{\mathbf{K}}_{0} \leftarrow \hat{\mathbf{K}}_{0} \backslash\{j\} ; / * \mathrm{RB}_{j}$ is no longer available, and should be removed from $\hat{\mathbf{K}}_{0} * /$

22. If $\left(v^{i}=0\right)$ set $\hat{\mathbf{M}} \leftarrow \hat{\mathbf{M}} \backslash\{i\} ; / * \mathrm{U}_{i}$ no longer needs $\mathrm{RBs}$, and should be removed from $\hat{\mathbf{M}} * /$

23. If $\left(\left(j \in \mathbf{K} \backslash\left(\bigcup_{n \in \mathbf{N}} \mathbf{K}_{n}\right) \cap \mathbf{K}_{0}\right)\right.$ or $\left.\left(u_{j}>1\right)\right)$ set $\left.\hat{\mathbf{K}} \leftarrow \hat{\mathbf{K}} \backslash\{j\} ;\right\} / *$ if $\mathrm{RB}_{j}$ belongs to the set of protected $\mathrm{RB}$ or has been allocated more than one time to the users, remove it from $\hat{\mathbf{K}} * /$

24. Else set $\hat{\mathbf{M}} \leftarrow \hat{\mathbf{M}} \backslash\{i\} ;\} / *$ If $\hat{\mathbf{K}}_{0}$ is empty, there are no more RBs that can be allocated in $\mathrm{K}_{0} * /$

25. Else $\left\{/ *_{\text {if }} U_{i}\right.$ is located within the service areas of one of the pNBs, it can access the available RBs of both the pNB and a macronetwork*/

26. $\quad$ For $(n \in \mathbf{N})\{$

27. If $\left(i \in \mathbf{M}_{n}\right)\{$

28. $\quad$ If $\left(\hat{\mathbf{K}}_{0} \cup \hat{\mathbf{K}}_{n} \neq \varnothing\right)\{$

29. $\quad$ Set $j \leftarrow \arg \min \left(\min _{k \in \hat{\mathbf{K}}_{n}}\left|\theta^{i}\left(v^{i}-\hat{r}_{k n}^{i}\right)\right|, \min _{k \in \hat{\mathbf{K}}_{0}}\left|\theta^{i}\left(v^{i}-\hat{r}_{k 0}^{i}\right)\right|\right)$;

30. If $\left(j \in \hat{\mathbf{K}}_{0}\right)\left\{\right.$ set $\left.\hat{\mathbf{K}}_{0} \leftarrow \hat{\mathbf{K}}_{0} \backslash\{j\}, y_{j}^{i} \leftarrow y_{j}^{i}+1, v^{i} \leftarrow\left\lceil v^{i}-\hat{r}_{j 0}^{i}\right\rceil^{+} ;\right\} / *_{\text {if }} \mathrm{RB}_{j}$ belongs to the spectrum of a macro-network, allocate RBs from $\hat{\mathbf{K}}_{0}$ to $\mathrm{U}_{i} * /$

31. $\quad$ Else $\left\{\right.$ set $\left.\hat{\mathbf{K}}_{n} \leftarrow \hat{\mathbf{K}}_{n} \backslash\{j\}, x_{j n}^{i} \leftarrow x_{j n}^{i}+1, v^{i} \leftarrow\left\lceil v^{i}-\hat{r}_{j n}^{i}\right]^{+} ;\right\} / *_{\text {if }} \mathrm{RB}_{j}$ belongs to the spectrum of a pNB , allocate $\mathrm{RBs}$ from $\hat{\mathbf{K}}_{n}$ to $\mathrm{U}_{i} * /$

32. $\quad$ Set $u_{j} \leftarrow u_{j}+1$;

33. If $\left(v^{i}=0\right)$ set $\hat{\mathbf{M}} \leftarrow \hat{\mathbf{M}} \backslash\{i\}$;

34. If $\left(\left(j \in \mathbf{K} \backslash\left(\bigcup_{n \in \mathbf{N}} \mathbf{K}_{n}\right) \cap \mathbf{K}_{0}\right)\right.$ or $\left.\left(u_{j}>1\right)\right)$ set $\left.\hat{\mathbf{K}} \leftarrow \hat{\mathbf{K}} \backslash\{j\} ;\right\}$

35. $\quad$ Else set $\hat{\mathbf{M}} \leftarrow \hat{\mathbf{M}} \backslash\{i\} ;\}\}\}\}$

36. Output $x^{m}{ }_{k n}, y^{m}{ }_{k}$, for all $n \in \mathbf{N}, m \in \mathbf{M}, k \in \mathbf{K}$. /*Output the results of RB allocation.*/ 


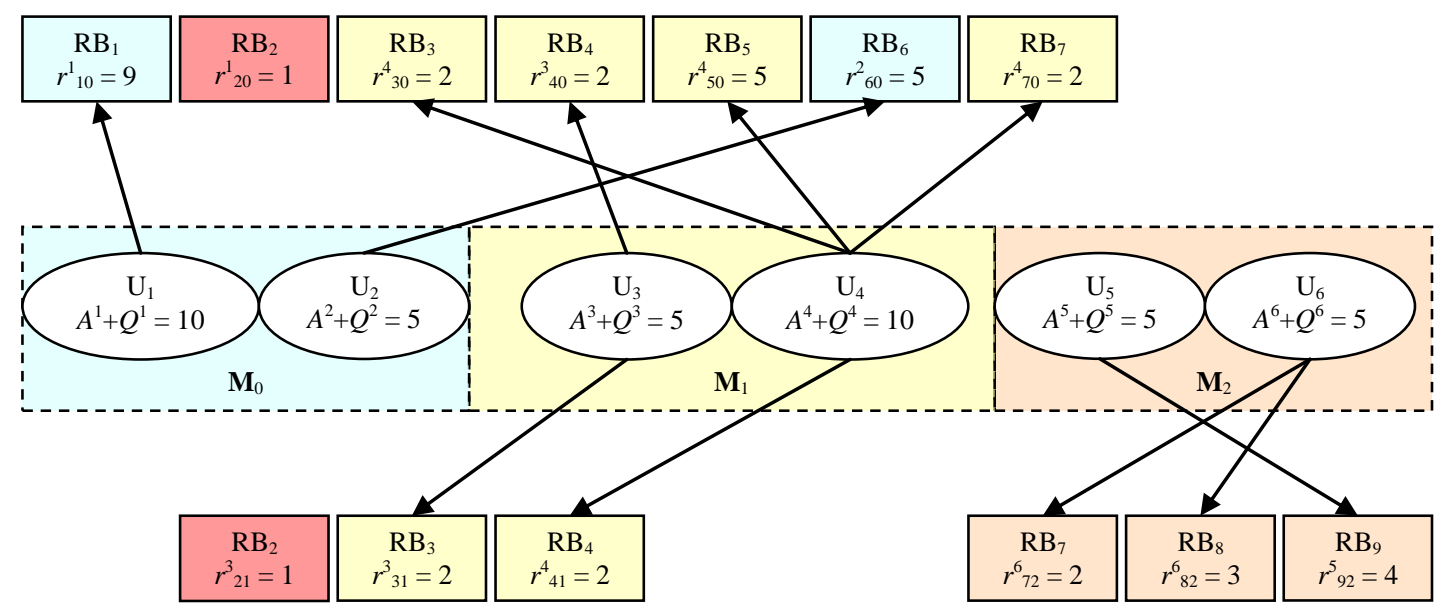

$N=2, M=6, K=9$

$\mathbf{M}=\{1,2,3,4,5,6\} ; \mathbf{M}_{0}=\{1,2\} ; \mathbf{M}_{1}=\{3,4\} ; \mathbf{M}_{2}=\{5,6\}$

$\mathbf{K}=\{1,2,3,4,5,6,7,8,9\} ; \mathbf{K}_{0}=\{1,2,3,4,5,6,7\} ; \mathbf{K}_{1}=\{2,3,4\} ; \mathbf{K}_{2}=\{7,8,9\}$

$\begin{array}{ccc}x^{m}{ }_{k 1} & m=1 & m=2 \\ k=1 & \mathbf{0} & \mathbf{0} \\ k=2 & \mathbf{0} & \mathbf{0} \\ k=3 & \mathbf{0} & \mathbf{1} \\ k=4 & \mathbf{0} & \mathbf{0} \\ k=5 & \mathbf{0} & \mathbf{0} \\ k=6 & \mathbf{0} & \mathbf{0} \\ k=7 & \mathbf{0} & \mathbf{0} \\ k=8 & \mathbf{0} & \mathbf{0} \\ k=9 & \mathbf{0} & \mathbf{0}\end{array}$

$\begin{array}{ccc}m=3 & m=4 & m=5 \\ \mathbf{0} & \mathbf{0} & \mathbf{0} \\ \mathbf{0} & \mathbf{0} & \mathbf{0} \\ \mathbf{1} & \mathbf{0} & \mathbf{0} \\ \mathbf{0} & \mathbf{1} & \mathbf{0} \\ \mathbf{0} & \mathbf{0} & \mathbf{0} \\ \mathbf{0} & \mathbf{0} & \mathbf{0} \\ \mathbf{0} & \mathbf{0} & \mathbf{0} \\ \mathbf{0} & \mathbf{0} & \mathbf{0} \\ \mathbf{0} & \mathbf{0} & \mathbf{0} \\ y^{m}{ }_{k} & m=1 & m=2 \\ k=1 & \mathbf{1} & \mathbf{0} \\ k=2 & \mathbf{0} & \mathbf{0} \\ k=3 & \mathbf{0} & \mathbf{0} \\ k=4 & \mathbf{0} & \mathbf{0} \\ k=5 & \mathbf{0} & \mathbf{0} \\ k=6 & \mathbf{0} & \mathbf{1} \\ k=7 & \mathbf{0} & \mathbf{0} \\ k=8 & \mathbf{0} & \mathbf{0} \\ k=9 & \mathbf{0} & \mathbf{0}\end{array}$

$m=6$
$\mathbf{0}$
$\mathbf{0}$
$\mathbf{0}$
$\mathbf{0}$
$\mathbf{0}$
$\mathbf{0}$
$\mathbf{0}$
$\mathbf{0}$
$\mathbf{0}$
$m=3$
$\mathbf{0}$
$\mathbf{0}$
$\mathbf{0}$
$\mathbf{1}$
$\mathbf{0}$
$\mathbf{0}$
$\mathbf{0}$
$\mathbf{0}$
$\mathbf{0}$

$x^{m}{ }_{k 2}$
$k=1$
$k=2$
$k=3$
$k=4$
$k=5$
$k=6$
$k=7$
$k=8$
$k=9$
$m=4$
$\mathbf{0}$
$\mathbf{1}$
$\mathbf{0}$
$\mathbf{0}$
$\mathbf{1}$
$\mathbf{0}$
$\mathbf{1}$
$\mathbf{0}$
$\mathbf{0}$

$\begin{array}{cc}m=1 & m=2 \\ \mathbf{0} & \mathbf{0} \\ \mathbf{0} & \mathbf{0} \\ \mathbf{0} & \mathbf{0} \\ \mathbf{0} & \mathbf{0} \\ \mathbf{0} & \mathbf{0} \\ \mathbf{0} & \mathbf{0} \\ \mathbf{0} & \mathbf{0} \\ \mathbf{0} & \mathbf{0} \\ \mathbf{0} & \mathbf{0} \\ m=5 & m=6 \\ \mathbf{0} & \mathbf{0} \\ \mathbf{0} & \mathbf{0} \\ \mathbf{0} & \mathbf{0} \\ \mathbf{0} & \mathbf{0} \\ \mathbf{0} & \mathbf{0} \\ \mathbf{0} & \mathbf{0} \\ \mathbf{0} & \mathbf{0} \\ \mathbf{0} & \mathbf{0} \\ \mathbf{0} & \mathbf{0}\end{array}$

$\begin{array}{cccc}m=3 & m=4 & m=5 & m=6 \\ \mathbf{0} & \mathbf{0} & \mathbf{0} & \mathbf{0} \\ \mathbf{0} & \mathbf{0} & \mathbf{0} & \mathbf{0} \\ \mathbf{0} & \mathbf{0} & \mathbf{0} & \mathbf{0} \\ \mathbf{0} & \mathbf{0} & \mathbf{0} & \mathbf{0} \\ \mathbf{0} & \mathbf{0} & \mathbf{0} & \mathbf{0} \\ \mathbf{0} & \mathbf{0} & \mathbf{0} & \mathbf{0} \\ \mathbf{0} & \mathbf{0} & \mathbf{0} & \mathbf{1} \\ \mathbf{0} & \mathbf{0} & \mathbf{0} & \mathbf{1} \\ \mathbf{0} & \mathbf{0} & \mathbf{1} & \mathbf{0}\end{array}$

Figure 4. Result of RB allocation in the network with $2 \mathrm{pNBs}$ and 6 users.

Now we are ready to present the proposed RB allocation procedure. A graphical illustration of applying this procedure to the small network with $2 \mathrm{pNBs}$ and 6 users is shown in Figure 4, where the RBs which do not satisfy the target SINR requirements (and, therefore, cannot be allocated to the users) are highlighted using red colour. According to this procedure, the all necessary calculations are performed by a central processor (CP) which collects all necessary information related to RB allocation. A CP could be installed either in one of the eNBs or in an LTE evolved packet core (EPC). In the first case (if $\mathrm{CP}$ is located in one of the eNBs), the information exchange between a $\mathrm{CP}$ and the eNBs/pNBs is realized via $\mathrm{X} 2$ interface. Otherwise (if $\mathrm{CP}$ is located in the EPC), the information exchange between a $\mathrm{CP}$ and the eNBs/pNBs is realized via a standard LTE S1 interface [1]. The following information is always available in the network (known to a $\mathrm{CP}$ and all the eNBs/pNBs):

- spectrum information $\mathbf{K}_{0}, \mathbf{K}_{1}, \ldots, \mathbf{K}_{N}$ (since each eNB/pNB operates on its fixed licensed spectrum band, there is no need to update this information);

- user location information $\mathbf{M}_{0}, \mathbf{M}_{1}, \ldots, \mathbf{M}_{N}$ (this information is gathered by the network through a standard RACH procedure [17] used for initial access to the network)

- the target SINR information, $\operatorname{SINR}^{m}{ }_{\text {tar }}$, and the user's weight $\theta^{m}$, for all $m \in \mathbf{M}$, can be updated periodically (e.g., once in an hour) by a CP based on the desired channel quality requirements of the users (obtained from all eNBs/pNBs).

The RB allocation procedure consists of the following steps (repeated at every slot $t$ ):

Step 1. Each user $\mathrm{U}_{m}$ sends its BSR data, $Q_{m}$ and $A_{m}$, and the recent CSI information, $\operatorname{SINR}^{m}{ }_{k 0}(t-1)$ and $\operatorname{SINR}^{m}{ }_{k n}(t-1)$, to the associated eNB/pNB via the dedicated PUCCH.

Step 2. All the eNBs/pNBs send the collected data to a $\mathrm{CP}$ via $\mathrm{X} 2$ or $\mathrm{S} 1$ interface.

Step 3. After collecting this data, a CP solves the problem (8a) - (8k) using the algorithm presented in Figure 3 and sends the optimal (near-optimal) RB allocation variables $x^{m}{ }_{k n}, y^{m}{ }_{k}$ to the respective eNBs/pNBs via X2 or S1 interface [1].

Step 4. Each eNB/pNB informs its users about the resource allocation decisions $x^{m}{ }_{k n}, y^{m}{ }_{k}$ via PDCCHs.

As follows from the above description, the proposed RB allocation procedure needs only two additional control 
signalling steps for its operation. The first additional signalling step is necessary to collect the data (using S1 or X2 interface) from the eNBs/pNBs; the second step is to inform the eNBs/pNBs about the resulted RB allocations. Hence, it is reasonable to suggest that the control overheads introduced by the proposed RB allocation procedure is minimal, and its impact on the network performance is neglectable.

\section{Performance Evaluation}

A simulation model of the network has been implemented upon a standard LTE-A Frequency Division Duplex (FDD) platform using the OPNET development package [20]. The model (illustrated in Figure 5) consists of the LTE EPC (with the integrated $\mathrm{CP}$ ), 2 eNBs (comprising a macro-network), and 3 pNBs. The EPC communicates with each eNB/pNB using the standard S1 interface via $10 \mathrm{Mbps}$ internet protocol (IP) links. Each eNB in the model has a service area with $5 \mathrm{~km}$ radius. Each pNB has a service area with $500 \mathrm{~m}$ radius. The service areas of the macrocells overlap with the service areas of the picocells but do not overlap with each other, and vice versa. The licensed bandwidth of each eNB equals $50 \mathrm{RBs}$ (which is equivalent to $10 \mathrm{MHz}$ in the frequency domain). Each pNB operates on the bandwidth comprising 25 RBs (or 5 $\mathrm{MHz}$ in the frequency domain). The simulated spectrum usage scenario is illustrated in Figure 6. Here the entire spectrum band of the $\mathrm{pNB}_{1}$ overlaps with the spectrum band of the $\mathrm{eNB}_{1}$. The bandwidth of the $\mathrm{pNB}_{2}$ overlaps partially with the spectrum band of the $\mathrm{eNB}_{1}$. The bands of the $\mathrm{eNB}_{2}$ and $\mathrm{pNB}_{3}$ are protected (since they do not overlap with the spectrum bands of other pNBs/eNBs).

The network serves $M$ UEs: $M / 5$ users located inside each picocell, the rest of the users are placed in the non-overlapping areas of a macro-network. The user traffic in simulations consists of three most frequently used network applications: Voice over IP (VoIP), video and Hyper-Text Transfer Protocol (HTTP). The number of users of each type is distributed in proportion 2:3:5 for voice, video, and data users, respectively. The following models (described in [35]) have been used to simulate voice, video and web users:

- The VoIP services model is ON-OFF model with exponentially distributed $\mathrm{ON}-\mathrm{OFF}$ periods. The mean duration of $\mathrm{ON}$ and $\mathrm{OFF}$ periods are $0.65 \mathrm{~s}$ and $0.352 \mathrm{~s}$, respectively. The VoIP traffic is generated by using the G.723.1 (12.2 Kbps) codec with a voice payload size 40 bytes and a voice payload interval $30 \mathrm{~ms}$.

- Video services are simulated using a high-resolution video model with a constant frame size equal 6250 bytes and exponentially distributed frame inter-arrival intervals (with mean equal $0.5 \mathrm{~s}$ ).

- Web users in simulations are HTTP1.1 users generating pages or images with exponential page inter-arrival intervals (mean equal $60 \mathrm{sec}$ ). It is assumed that one page consists of one object, whereas one image consists of five objects. The object size is constant and equal 1000 bytes.

A radio model of the network has been developed according to the ITU-T Recommendation M.1225. It is assumed that the users operate in a moderately dense urban environment. The desired target SINR levels equal $\operatorname{SINR}^{m}{ }_{\text {tar }}=0 \mathrm{~dB}$, for all $m \in \mathbf{M}$. The weights of the users are set as $\theta^{m}=1$, for all voice and video users and $\theta^{n}=0$, for all HTTP users. The maximal transmission power levels of the eNB, pNB and UE are $P_{e N B}=$ $20 \mathrm{~W}, P_{p N B}=2 \mathrm{~W}$ and $P_{U E}=200 \mathrm{~mW}$, respectively. Other network parameters are set in accordance with the requirements of the LTE specifications [1] (the simulation parameters of the model are listed in Table I).

First, we evaluate the complexity and efficiency (in terms of finding the optimal solution) of different methods used to solve the problem $(9 a)-(9 k)$. The first method is the version of an exact B\&B algorithm (described in [28]) deployed to speed up the solution process. We call this method Exact $B \& B$ (abbreviated EB\&B). The second method is the application of a GA approach (outlined in Figure 3) proposed in this paper to find a suitable (but not necessarily optimal) solution. We call this method Modified GA (abbreviated MGA). Tables 2 and 3 and Figure 6 summarize the performance of the algorithms, with $M$ ranging from 20 to 400 users. Table 2 shows the maximal, average and minimal number of iterations in both algorithms. Table 3 presents the maximal, average and minimal solution time (in $\mu \mathrm{s}$ ). Figure 7 illustrates the percent of optimal solutions in MGA algorithm, and the mean absolute percentage deviation (MAPD) between a solution and an optimal result. Here the MAPD is calculated using [36]:

$M A P D=\frac{100 \%}{T} \sum_{t=1}^{T}\left|\frac{O_{t}-S_{t}}{O_{t}}\right|, O_{t}=\sum_{m \in \mathbf{M}} \bar{R}^{m}, S_{t}=\sum_{m \in \mathbf{M}} R_{M G A}^{m}$,

where $T$ is the total number of simulation trials; $O_{t}$ is the value of the objective function in a trial $t$ at the optimal point, $S_{t}$ is the value of the objective function in a trial $t$ at the solution point found using the proposed MGA algorithm. Results of simulations demonstrate that the number of iterations and solution time in MGA algorithm is at least 4 times smaller than those in EB\&B algorithm. Although MGA may fail to obtain the optimal solution, the probability of such a failure in MGA is rather small $(<3 \%)$ with the relatively low MAPD (< $1 \%)$. The solution time in EB\&B might exceed $T_{s}=0.5 \mathrm{~ms}$ (slot duration in LTE system). Hence, in some cases, the EB\&B algorithm will not produce the optimal result in due time (i.e. within the $0.5 \mathrm{~ms}$ long resource allocation period).

We now compare the performance of the proposed MGA and $\mathrm{EB} \& \mathrm{~B}$ with the performance of the following (most relevant to the considered scenario) resource allocation schemes:

- The first scheme (proposed in [37]) has been designed for energy-efficient power allocation in the integrated pico/macrocell LTE-A network. Here the total service rate in picocells is maximized subject to the target SINR constraints (more comprehensive description of this scheme can be found in [37]).

- In the second scheme (presented in [38]), the RBs are allocated to the users of the picocells to maximize the global proportional fairness in the network. The corresponding optimization problem is NP-hard and, hence, instead of solving the problem, the authors propose a simple user association rule to distribute the resources. 


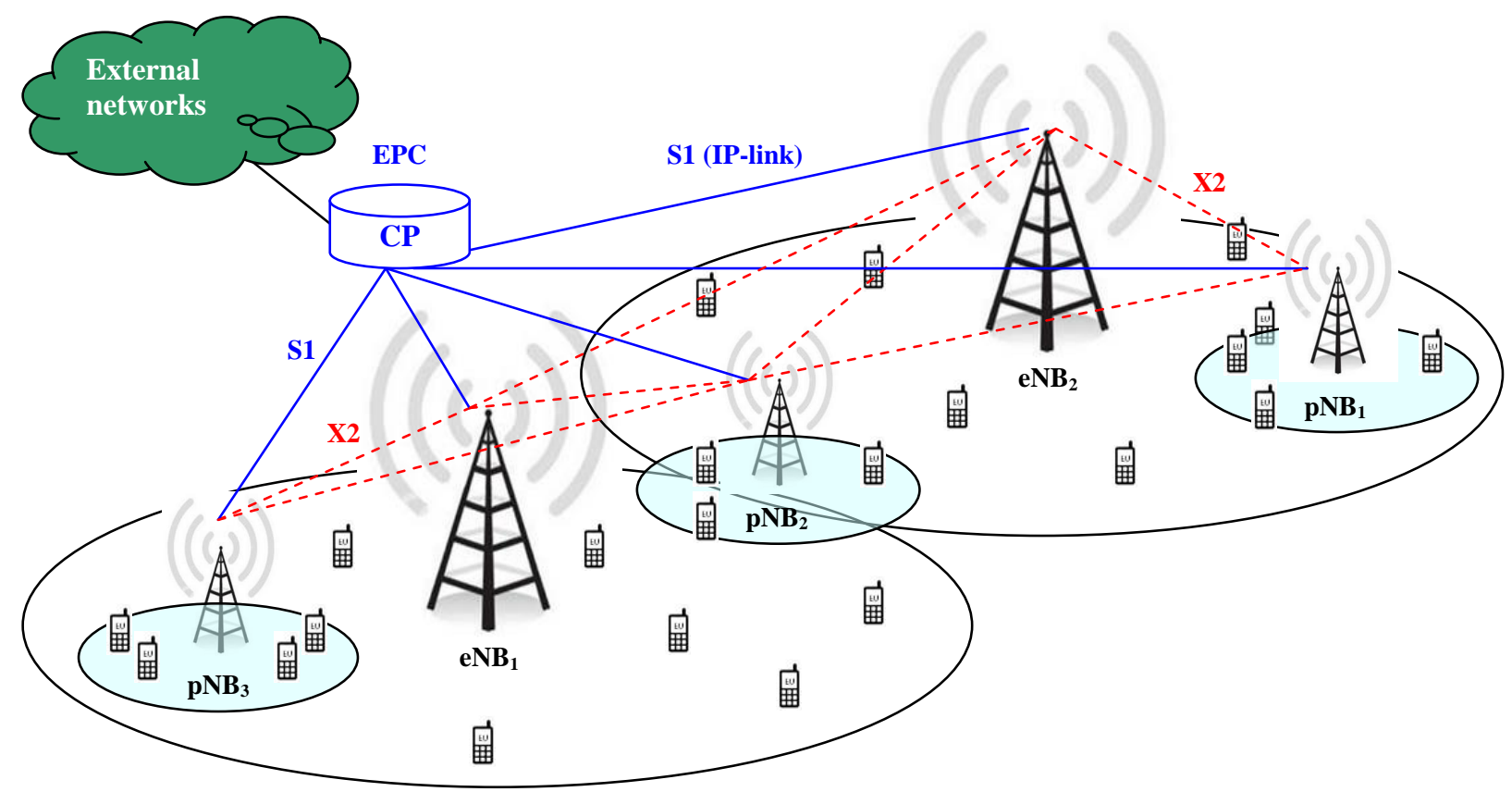

Figure 5. The simulation model of a network comprising $2 \mathrm{eNBs}, 3 \mathrm{pNBs}$ and EPC with an integrated CP.

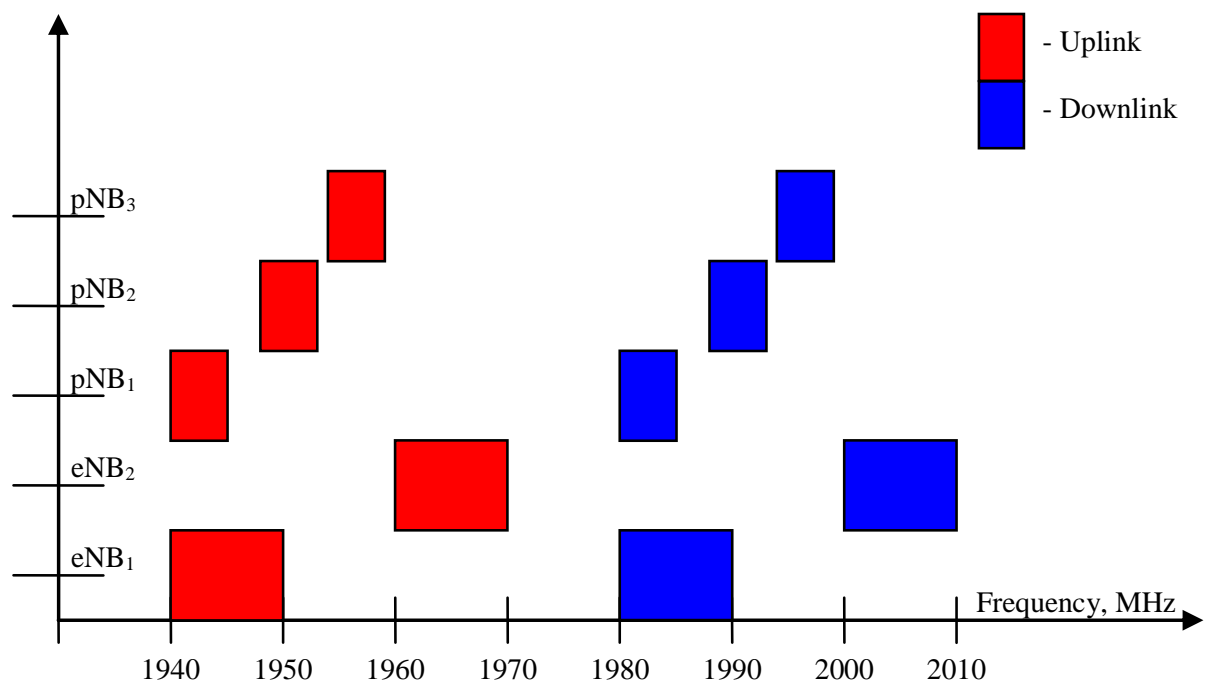

Figure 6. The UL and DL frequency bands of the eNBs/pNBs in simulation model.

TABLE I. SIMULATION PARAMETERS OF THE MODEL

\begin{tabular}{|c|c|c|}
\hline \multicolumn{2}{|c|}{ Parameter } & Value \\
\hline \multirow[t]{10}{*}{ Radio Network Model: } & Path loss & $L=40 \log _{10} R+30 \log _{10} f+49, R-$ distance $(\mathrm{km}), f-$ carrier frequency $(\mathrm{Hz})$ \\
\hline & Shadow fading & $\begin{array}{l}\text { Log-normal shadow fading with a standard deviation of } 10 / 12 \mathrm{~dB} \text { for outdoor/indoor } \\
\text { users }\end{array}$ \\
\hline & Penetration loss & The average building penetration loss is $12 \mathrm{~dB}$ with a standard deviation of $8 \mathrm{~dB}$ \\
\hline & Multipath fading & Spatial Channel Model (SCM), Suburban macro \\
\hline & UE velocity & $0 \mathrm{~km} / \mathrm{s}$ \\
\hline & Transmitter/Receiver antenna gain & $10 \mathrm{dBi}$ (pedestrian), $2 \mathrm{dBi}$ (indoor) \\
\hline & Receiver antenna gain & $10 \mathrm{dBi}$ (pedestrian), $2 \mathrm{dBi}$ (indoor) \\
\hline & Receiver noise figure & $5 \mathrm{~dB}$ \\
\hline & Thermal noise density & $-174 \mathrm{dBm} / \mathrm{Hz}$ \\
\hline & Cable/connector/combiner losses & $2 \mathrm{~dB}$ \\
\hline \multirow[t]{4}{*}{ Physical profile: } & Operation mode & FDD \\
\hline & Cyclic Prefix Type & Normal (7 Symbols per Slot) \\
\hline & EPC Bearer Definitions & 348kbit/s (Non-GBR) \\
\hline & Subcarrier spacing & $15 \mathrm{kHz}$ \\
\hline Admission Control & PDCCH symbols per subframe & 3 \\
\hline
\end{tabular}




\begin{tabular}{|c|c|c|}
\hline \multirow[t]{3}{*}{ Parameters: } & UL Loading Factor & 1 \\
\hline & DL Loading Factor & 1 \\
\hline & Inactive Bearer Timeout & $20 \mathrm{sec}$ \\
\hline \multirow[t]{2}{*}{ BSR Parameters: } & Periodic Timer & 5 subframes \\
\hline & Retransmission Timer & 2560 subframes \\
\hline \multirow[t]{4}{*}{ L1/L2 Control Parameters: } & Reserved Size & $2 \mathrm{RBs}$ \\
\hline & Cyclic Shifts & 6 \\
\hline & Starting RBP for Format 1 messages & 0 \\
\hline & Allocation Periodicity & 5 subframes \\
\hline \multirow[t]{6}{*}{ Random Access Parameters: } & Number of Preambles & 64 \\
\hline & Preamble Format & Format 0 (1-subframe long) \\
\hline & Number of RA Resources per Frame & 4 \\
\hline & Preamble Retransmission Limit & 5 subframes \\
\hline & RA Response Timer & 5 subframes \\
\hline & Contention Resolution Timer & 40 subframes \\
\hline \multirow[t]{3}{*}{ HARQ Parameters: } & Maximal Number of Retransmissions & 3 (uplink and downlink) \\
\hline & HARQ Retransmission Timer & 8 subframes (uplink and downlink) \\
\hline & $\begin{array}{l}\text { Maximal Number of HARQ } \\
\text { processes }\end{array}$ & 8 per UE (uplink and downlink) \\
\hline
\end{tabular}

TABLE II. NUMBER OF ITERATIONS IN EB\&B AND MGA ALGORITHMS

\begin{tabular}{c|r|r|r|r|r|r}
\hline \hline \multirow{2}{*}{ Number of users, $M$} & \multicolumn{3}{|c|}{ EB\&B } & \multicolumn{3}{c}{ MGA } \\
\cline { 2 - 7 } & Min & Avg & Max & Min & Avg & Max \\
\hline 20 & 4 & 23 & 69 & 3 & 5 & 10 \\
\hline 40 & 5 & 53 & 161 & 4 & 11 & 21 \\
\hline 60 & 4 & 84 & 257 & 4 & 19 & 34 \\
\hline 80 & 4 & 117 & 348 & 4 & 26 & 47 \\
\hline 100 & 5 & 150 & 452 & 4 & 33 & 61 \\
\hline 120 & 5 & 185 & 567 & 4 & 41 & 74 \\
\hline 140 & 6 & 221 & 675 & 5 & 49 & 89 \\
\hline 160 & 6 & 257 & 770 & 5 & 57 & 102 \\
\hline 180 & 6 & 293 & 893 & 4 & 65 & 117 \\
\hline 200 & 5 & 331 & 995 & 5 & 74 & 133 \\
\hline 220 & 5 & 368 & 1107 & 5 & 81 & 148 \\
\hline 240 & 5 & 406 & 1220 & 5 & 90 & 162 \\
\hline 260 & 5 & 444 & 1335 & 5 & 99 & 178 \\
\hline 280 & 5 & 483 & 1451 & 5 & 107 & 195 \\
\hline 300 & 6 & 522 & 1571 & 5 & 115 & 208 \\
\hline 320 & 5 & 561 & 1683 & 5 & 125 & 225 \\
\hline 340 & 5 & 601 & 1803 & 5 & 133 & 241 \\
\hline 360 & 6 & 640 & 1934 & 5 & 142 & 256 \\
\hline 380 & 6 & 681 & 2051 & 6 & 152 & 272 \\
\hline 400 & 5 & 721 & 2169 & 5 & 160 & 288 \\
\hline \hline
\end{tabular}

TABLE III. SOLUTION TIME ( $\mu$ s) IN EB\&B AND MGA ALGORITHMS

\begin{tabular}{c|r|r|r|r|r|r}
\hline \hline \multirow{2}{*}{ Number of users, $M$} & \multicolumn{3}{|c|}{ EB\&B } & \multicolumn{3}{|c}{ MGA } \\
\cline { 2 - 7 } & Min & Avg & Max & Min & Avg & Max \\
\hline 20 & 3 & 7 & 27 & 2 & 4 & 2 \\
\hline 40 & 4 & 10 & 53 & 3 & 6 & 5 \\
\hline 60 & 5 & 17 & 86 & 3 & 6 & 7 \\
\hline 80 & 4 & 23 & 121 & 3 & 6 & 11 \\
\hline 100 & 4 & 30 & 151 & 2 & 5 & 14 \\
\hline 120 & 4 & 37 & 186 & 3 & 7 & 17 \\
\hline 140 & 4 & 44 & 224 & 3 & 8 & 20 \\
\hline 160 & 5 & 51 & 258 & 3 & 9 & 23 \\
\hline 180 & 4 & 59 & 296 & 4 & 11 & 27 \\
\hline 200 & 5 & 66 & 332 & 3 & 12 & 30 \\
\hline 220 & 5 & 74 & 370 & 3 & 13 & 34 \\
\hline 240 & 5 & 81 & 409 & 3 & 15 & 37 \\
\hline 260 & 5 & 89 & 446 & 3 & 16 & 41 \\
\hline 280 & 5 & 97 & 483 & 3 & 18 & 44 \\
\hline 300 & 5 & 104 & 524 & 3 & 19 & 48 \\
\hline 320 & 5 & 112 & 563 & 3 & 21 & 51 \\
\hline 340 & 6 & 120 & 603 & 4 & 22 & 55 \\
\hline 360 & 6 & 128 & 642 & 3 & 23 & 59 \\
\hline 380 & 5 & 136 & 685 & 3 & 25 & 62 \\
\hline 400 & 5 & 144 & 722 & 3 & 26 & 66 \\
\hline \hline
\end{tabular}

Further in the paper, the first and second schemes will be called RA1 (resource allocation procedure 1) and RA2 (resource allocation procedure 2), respectively.

The graphs in Figures $8-10$ illustrate the performance of different resource allocation methods in the experiments with varying number of users. All algorithms are simulated with identical system parameters. Figure 8 shows the mean SINR in the UL and DL channels between the users and the pico/macro-network. Figure 9 demonstrates the mean medium access layer (MAC) throughput in the UL and DL channels between the users and the pico/macro-network. Figure 10 shows the mean packet end-to-end delay for the users. It follows from these figures that the highest SINR levels are achieved by RA1 (which is a rather expected outcome, because in this scheme the power allocated to the channels of the picocells is constrained by the target SINR levels in these picocells [37]). Note, however, that RA1 is not very good in terms of maintaining the throughput and reducing delay for the network users. The reason behind such poor performance is that some users are allocated with very low power levels (to satisfy the target SINR constraints) which result in low service rate and consequently, low throughput and large delays for the users. RA2 shows the worst performance in terms of SINR and slightly better (than RA1) performance in terms of throughput and delay. Note that in this scheme, instead of directly solving the formulated optimization problem, the user association rule is used to allocate the RBs [38]. Hence, although the proposed user association rule is very simple, it does not guarantee optimal distribution of spectrum resources. As a result, the performance of RA2 for the users is not very good. Both EB\&B and MGA algorithms achieve satisfactory (better than RA2 and slightly worse than RA1) SINR and good (better than RA1 and RA2) throughput and delay performance. Note that both of these algorithms use formulation $(9 a)-(9 k)$ for RB allocation with the objective to minimize the future queue size in the buffers of UEs (leading to reduced delay for UEs). Although MGA does not directly solve the problem (9), it has very high probability of finding the optimal solution (or providing the solution which is very close to optimal, as it follows from Figure 7). Therefore, both 
$\mathrm{EB} \& \mathrm{~B}$ and MGA are very effective in increasing the throughput and reducing delay for the network users while maintaining reasonably high (not lower than the target) SINR levels in the wireless channels.

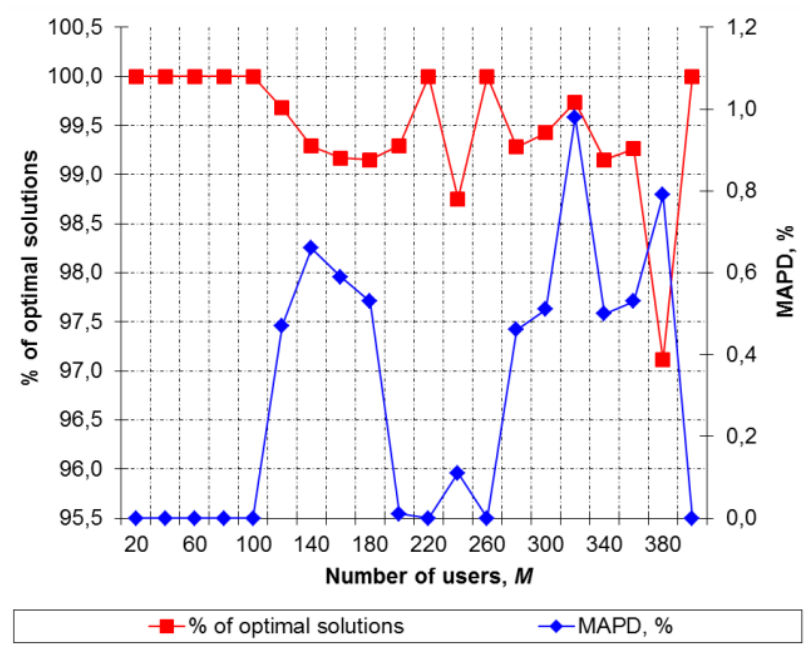

Figure 7. Percent of found optimal solutions and MAPD (\%) between solution obtained in MGA and optimal result.

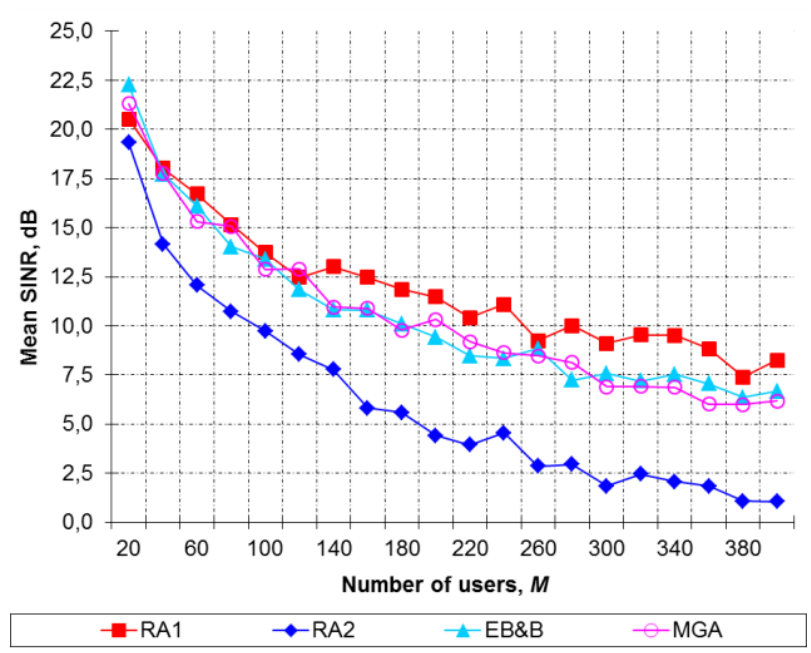

Figure 8. Mean SINR in different resource allocation schemes.

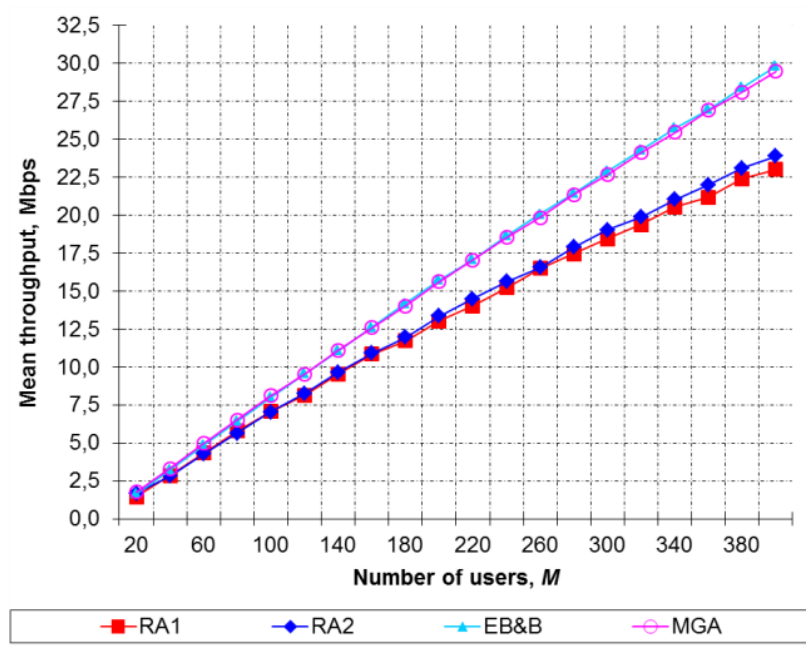

Figure 9. Mean throughput in different resource allocation schemes.

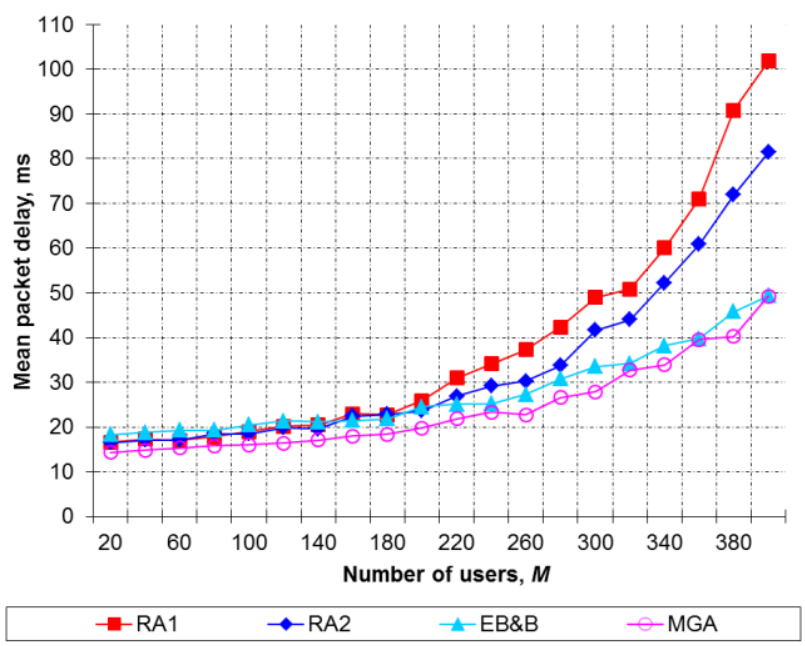

Figure 10. Mean packet and-to-end delay in different resource allocation schemes.

To conclude, we note that both the exact $(\mathrm{EB} \& \mathrm{~B})$ and heuristic (MGA) algorithms show very similar performance for the users. However, because of its reduced complexity and smaller solution time, the latter algorithm (i.e., MGA) is preferable for use in practical network deployments.

\section{CONCLUSION}

In this paper, we propose the novel approach for RB allocation in the integrated picocell/macrocell LTE-A network. The main idea here is to use the queue size in the buffers of UEs as a network utility. The formulated RB allocation problem belongs to the family of MKPs (which are NP-hard in the strong sense). Consequently, we show two possible ways to deal with this problem: i) applying the exact branch-and-bound (B\&B) technique to speed up the solution process, ii) utilizing the modified GA heuristics to find suitable (but not necessarily optimal) solution. The RB allocation procedure derived in the paper use both of the above algorithms (exact and heuristic) for RB allocation. Both algorithms show good performance in simulations. However, the approximate algorithm has much lower complexity and smaller solution time than the exact method and, therefore, it is more suitable for practical network deployments.

\section{REFERENCES}

[1] Evolved Universal Terrestrial Radio Access (E-UTRA) and Evolved Universal Terrestrial Radio Access Network (E-UTRAN); Overall Description. 3GPP TS 36.300. V11.3.0.

[2] J. Damnjanovic et. al., A survey on 3GPP heterogeneous networks, IEEE Wireless Communication, Vol. 18 (3), 2011, pp. 10-21.

[3] Service requirements for Home Node B (HNB) and Home eNode B (HeNB). 3GPP TS 22.220. Release 12.

[4] D. Lopez-Perez et. al., Enhanced intercell interference coordination challenges in heterogeneous networks, IEEE Wireless Communication, Vol. 18 (3), 2011, pp. 22-30.

[5] X. Y. Wang, P.-H. Ho and K.-C. Chen, Interference analysis and mitigation for cognitive-empowered femtocells through stochastic dual control, IEEE Transaction on Wireless Communication, Vol. 11 (6), 2012, pp. 2065-2075.

[6] Y.-S. Liang et. al., Resource allocation with interference avoidance in OFDMA femtocell networks, IEEE Transaction on Vehicular Technology, Vol. 61 (5), 2012, pp. 2243-2255. 
[7] Y. Sun, R.P. Jover, X. Wang, Uplink interference mitigation for OFDMA femtocell networks, IEEE Transaction on Wireless Communication, Vol. 11 (2), 2012, pp. 614-625.

[8] Ö. Bulakci et. al., Impact of power control optimization on the system performance of relay based LTE-advanced heterogeneous networks, Journal of Communication and Networking, Vol. 13 (4), 2012, pp. 345359.

[9] R. Combes, Z. Altman, E. Altman, Self-organizing relays: dimensioning, self-optimization, and learning, IEEE Transaction on Network and Service Management, Vol. 9 (4), 2012, pp. 487 - 500.

[10] T. Kudo and R. Ohtsuki, Cell range expansion using distributed $Q$ learning in heterogeneous networks, EURASIP Journal on Wireless Communications and Networking, Vol. 2013 (61), 2013.

[11] A. Daeinabi, K. Sandrasegaran and X. Zhu, Performance Evaluation of Cell Selection Techniques for Picocells in LTE-Advanced Networks, In Proc. IEEE ECTI-CON, 2013, pp. 1 - 6.

[12] B. Shrestha, E. Hossain, and K. W. Choi, An Analysis of Wireless Backhaul for Picocell Base Stations in Heterogeneous Networks, In Proc. IEEE Globecom, 2013, pp. 4927 - 4932.

[13] Y. Yu and D. Gu, The Cost Efficient Location Management in the LTE Picocell/Macrocell Network, IEEE Communications Letters, Vol. 17 (5), 2013, pp. 904-907.

[14] N. Obaid, A. Czylwik, An Efficient Macrocell Power Control Algorithm for Enhancing Dense Picocell Deployments, In Proc. European Wireless Conference, 2014, pp. 1-6.

[15] S. Kumar, S. Kalyani, and K. Giridhar, Spectrum Allocation For ICIC Based Picocell, accepted in IEEE Transactions On Vehicular Technology, 2014.

[16] T. Mimura et. al., Fixed-Rate Resource Exchange for Multi-Operator Pico eNodeB, IEICE Transactions on Communications; Vol.2013 (11), 2013, pp.2913-2922.

[17] LTE for UMTS: Evolution to LTE-Advanced. By H. Holma and A. Toskala, John Wiley and Sons, 2011.

[18] E-UTRA; MAC protocol specification. 3GPP TS 36.321. Release 8.

[19] Algorithms for Knapsack Problems. By D. Pisinger. Ph.D. thesis, DIKU, University of Copenhagen, Report 95/1, 1995.

[20] OPNET. [Online]. Available: http://www.opnet.com/

[21] Evolved Universal Terrestrial Radio Access Network (E-UTRAN); X2 Application Protocol (X2AP). 3GPP TS 36.423. Release 11.

[22] Physical Channels and Modulation. 3GPP TS 36.211. Release 8.

[23] Multiplexing and Channel Coding. 3GPP TS 36.212. Release 8.

[24] D. V. Lindley. The theory of queues with a single server. Math. Proc. of the Cambridge Philosophical Society, Vol. 48 (2), 1952, pp. 277-289.

[25] Enumerative Approaches to Combinatorial Optimization - Part 2, Annals of Operations Research, 11, 1987.

[26] S. Martello, P. Toth, A Bound and Bound algorithm for the zero-one multiple knapsack problem, Discrete Applied Mathematics, Vol. 3 (4), 1981, pp. 275-288.

[27] M. Visée et. al., Two-phases Method and Branch and Bound Procedures to Solve the Bi-objective Knapsack Problem, Journal of Global Optimization, Vol. 12 (2), 1998, pp 139-155.

[28] D. Pisinger, An exact algorithm for large multiple knapsack problems, European Journal of Operational Research, Vol. 114 (3), 1999, pp. 528 541.

[29] S. Martello, P. Toth, Heuristic algorithms for the multiple knapsack problem, Computing, Vol. 27 (2), 1981, pp 93-112.

[30] S. Khuri, T. Bäck and J. Heitkötter, The zero/one multiple knapsack problem and genetic algorithms, In Proc. ACM Symposium on Applied Computing, 1994, pp. 188-193.

[31] C. Cotta, J. M. Troya, A Hybrid Genetic Algorithm for the 0-1 Multiple Knapsack Problem, Artificial Neural Nets and Genetic Algorithms, 1998, pp. 250-254.

[32] M. E. Lalami et. al., A procedure-based heuristic for 0-1 Multiple Knapsack Problems, Int. J. Mathematics in Operational Research, Vol. 4 (3), 2012, pp. $214-224$.

[33] A.H.G. Rinnooy Kan, L. Stougie and C. Vercellis, A class of generalized greedy algorithms for the multi-knapsack problem, Discrete Applied Mathematics, Vol. 42 (2-3), 1993, pp. 279-290.

[34] M. J. Neely and R. Urgaonkar, Optimal Backpressure Routing in Wireless Networks with Multi-Receiver Diversity, Ad Hoc Networks, Vol. 7 (5), 2009, pp. $862-881$.

[35] IPOGUE, Internet study 2007 and 2008/2009, research report.

[36] H. Y. Yamin, S. M. Shahidehpour, Bidding Strategies Using Price Based Unit Commitment in a Deregulated Power Market, Electric Power Components and Systems, Vol. 32 (3), 2004, pp. 229-245.
[37] Y. Fu et. al., An Energy-Efficient Dense PicoStation Deployment and Power Control Strategy for Heterogeneous Networks, Smart Computing Review, Vol. 3 (1), 2013, pp. 24 - 32.

[38] D. Fooladivanda and C. Rosenberg, Joint Resource Allocation and User Association for Heterogeneous Wireless Cellular Networks, IEEE Transactions on Wireless Communications, Vol. 12 (1), 2012, pp. 248 257. 